II.

\title{
Die alte Frankfurter Deutsche Uebersetzung der Goldenen Bulle Kaiser Karls IV.
}

\author{
Von \\ Bibliothekar und Privatdocent Dr. Wilh. Altmann \\ in Greifswald.
}

Wo sind die Zeiten hin, da die genaue Kenntniss der Goldenen Bulle Kaiser Karls IV. als unbedingt erforderlich galt! Heute sind insbesondere die Studirenden der Geschichte und Rechtswissenschaft zu zählen, welche dieses so überaus wichtige Reichsgesetz einmal gelesen haben. Als erschwerendes Moment für das Studium der G. B. scheint von Vielen der Umstand angesehen zu werden, dass sie in lateinischer Sprache geschrieben ist; aus diesem Grunde hat wohl seinerzeit auch eine deutsche Uebersetzung der G. B. in die Sammlungen der Reichsabschiede (Corpus recessuum) Aufnahme gefunden, wie auch deutsche Uebersetzungen in Handschriften und gedruckten Ausgaben des 15.-18. Jahrhunderts zahlreich vorhanden sincl. Eine handliche neuere Ausgabe einer Uebersetzung der G. B. besitzen wir aber nicht. Der Wunsch, eine solche bei historischen Uebungen mit der lateinischen Sprache Nichtkundigen zu Grunde zu legen, bestimmte mich zur Heransgabe des folgenden Textes; denn eine moderne deutsche Uebersetzung zu geben erschien mir aus vielen Gründen unangebracht.

Da eine officielle gleichartige deutsche Uebersetzung der G. B. nicht existirt und auch nie existirt hat, so konnte es für mich nicht $z$ weifelhaft sein, dass ich die älteste unter den zahlreichen Uebersetzungen zum Abdruck zu bringen hatte. Als solche muss nach den eingehenden Untersuchungen Harnacks (Das Kurfürstencollegium bis zur Mitte des 14. Jahrhunderts S. 182 ff.), auf welche ich hier verweisen kann, die im Frankfurter Stadtarchive befindliche angesehen werden, welche 
in der äusseren Form den Originalausfertigungen sehr ähnlich ist, aber, da sie unbesiegelt ist, keine officielle Geltung beanspruchen kann.

Wenn Harnack nach Mittleilungen des damaligen frankfurtischen Stadtarchivars versichern konnte, dass die Handschrift dieser Uebersetzung diejenige eines in den siebziger Jahren des 14. Jahrhunderts noch häufig bemerkbaren Frankfurter Schreibers ist und somit nicht aus der Reichskanzlei stammt, so glaube ich unten bei Cap. 19 nachweisen zu können, dass diese Uebersetzung spätestens vor Mai 1365 entstanden sein muss; auch liegt ibr nicht, wie die Capitelüberschriften erkennen lassen, das Exemplar der G. B. zu Grunde, welches die Stadt Frankfurt im Jahre 1366 vom Kaiser erworben hat.

Der folgende Abdruck schliesst sich aufs Engste an die Handschrift ${ }^{1}$ an, doch sind grosse Anfangsbuchstaben nur nach Punkten gesetzt, $i$ und $j, u$ und $v$, welche in der Hds. willkürlich durcheinandergehen, geschieden worden. Betreffs der Interpunction, Satzabtheilung u. s. w. folge ich der Ausgabe in: „Ausgewählte Urkunden zur Erläuterung der Verfassungsgeschichte Deutschlands im Mittelalter. Herausgegeben von Wilh. Altmann und E. Bernheim. 2. Auflage. 1895."

Da der Uebersetzer die vielen eingeschalteten Sätze und Participialconstructionen des Originals vielfach, auch durch Umstellungen, zu vermeiden gesucht hat, so ist die Uebersetzung oft viel deutlicher und erleichtert mitunter die Interpretation; möchte sie in den Kreisen, an die ich bei der Herausgabe gedacht habe, Eingang finden.

\section{[I. Theil. Die Nürnberger Gesetze.]}

In $^{2}$ namen der heiligin undeilsamen drivaltekeit seleclich amen. Karle der firde mit gunste gotlichir miltekeit Romischir keisir allezit merer und konig zü Beheim. Des dingis zů

1 Es ist mitunter zweifelhaft, ob nicht statt $t z$, wie ich immer drucke, cz zu lesen ist; ebenso kann $\stackrel{\circ}{\mathrm{u}}$ mitunter auch für $\stackrel{\mathrm{u}}{\mathrm{u}}$ gehalten werden. Das tt der Hds, sieht einem ck sehr ähnlich. - ${ }^{2}$ Die 14 Hexameter, welche der Originalfassung der G. B. vorausgehen, boten dem Uebersetzer solche Schwierigkeiten, dass er sie wegliess; doch stehen sie lateinisch auf der letzten Seite unserer Hds. (S. 72), von anderer Hand geschrieben. Weggelassen ist in der Uebersetzung auch die Zusammenstellung der Capitelüberschriften des I. Theiles. 
ewegem gedenckin. Ein iegelich rich, daz in yme selbir zurdeilit ist, daz wird zurstorit; wan sine fursten sint wordin der diebe gesellin, darumb hat got mittin undir sie gemischit eynen swindeldin geist, daz sie stalpin ${ }^{1}$ an dem mittem dage alse in dem finstern, und hat ir kirtzstal ${ }^{2}$ bewegit von siner stat, und sint blint und furer der blinden; und wer in den finstern get, der stossit sich, und mit blindem gedancke begent sie vil meindat, die in der deilünge geschehint. Sage, do̊ hochfard, wye mochtest du an Lucefer han gerichtzit, hettest dů zurdrennůnge nit zi̊ helfe gehabit? Sage, dů nideger dufil, wie hettest d̊̊ Adam uz dem paradyse geworffin, hettest di̊ in nit von gehorsam gescheiden? Sage, dủ unkuschekeit, wie mochtest du Throya han gestorit, hettest dů frauwe Lenen nit von erem manne gescheidin? Sage, zorn, wie kundest du daz Romische gemeyne gut $^{3}$ zŭstorit han, hedest du nit von der zweyůnge Pompii und Julii zusamene gereißit den strit mit scharpin swertin bit uf daz gedirme? So̊ndir ${ }^{*}$ du, haz cristinlichis keisirdums, daz von gode ist gestirckit glich der heiligin undeillichin drivaltikeit mit den gotlichin dogenden glaube zůvirsicht und minne, des fullemůnt ${ }^{5}$ uff daz allircristenlichiste rich seleclich ist befestint, hastu dich mit dimre altin virgift als ein slange meyndedeclichin bekotzit an die keisirliche winrebe und an die nesten gledir des keisirdummys, so dye sule zuquetzschit weren, daz dủ den bu des keisirdůmis zủmale fellig mechtist; manigfelteclich hastu zweyunge geleit undir die sobin kurfursten des heiligin richis, durch dje als durch sebin luchtinde kirtzluchtir daz heilige keisirdům sal herluchtit werdin in eynekeit des sebingebinden ${ }^{6}$ geistis. In drůen ${ }^{7}$ wan ${ }^{8}$ wir von amptes wegin, von dem wir gebruchen keisirliche wirdekeit, schuldig sin zů begegen kunftigem schadin der zurdeilunge missehelle undir den kurfursten, in der zal wir auch [sint] alse ein konig von Beheim, von zweyn sachin, beide von keisirdim und von recht der kure, der wir gewonlichin nutzen: so han wir in unserm uffin hofe zů Nurenberg, da alle kurfursten geistlich und werntlich by uns sassin mit zallichir menige andern fursten grefin baneren frien edelen

1 stalpin (lat. palpent) stampfen, stolpern. $-{ }^{2}$ Gestell für Leuchter; Leuchter. - ${ }^{3}$ rem publicam. - + Tu quidem, invidia, christianum imperium $!-{ }^{5}$ fundamentum. $-{ }^{\circ}$ septiformis. $-{ }^{7}$ Sane cum $-{ }^{8}$ Hds. von 
und der stede uf dem stule keisirlichir almechtekeit, gezerit mit keisirlichir ymfeln wat ${ }^{1}$ und krone, mit sedegemme båradin můde von fullenkomenheit keisirlichis gewaltis gemacht gesetzit und mit keisirlichem bekanteniße bestedigit die nachgeschrebenen gesetze, recht zů schuren ${ }^{2}$ und zů fưren einbirkeit undir den kurfursten und ein eynmige wal inzulegene und auch zů furbeslißin den zůgang der vorgenant virsmehit zurdrennůnge und manigirhande schadin, die daruz nochfulgent. Dis ist geschehin ${ }^{3}$ anno domini 1356 indiccione nona 4 . idus januarii in deme zehinden jare unsir riche und in deme irstin jare des keisirdummys.

Wie daz geleit sulle sin der kurfursten. Capit. primum.

[1] Wir setzin von sichirm wißen von fullenkommener keisirlichir gewalt und bestedegen mit desem keisirlichim gebode, so wanne und wie dickewie hienach notdurfft uffstet in geschichte, ein Romischin konig z⿺̊̊ welin zủ eyme kunfftigen keisir, und die kurfursten nach altir labelichir gewonheit zin der kur sullin farin, so ist ein iegelichir kurfurste, ob iz und wan iz an in gemudit ${ }^{4}$ wird, schuldeg $z$ i geleidin eynen iegelichin sinen medekurfursten adir ir boten, die sie darzu sendent, durch ir lant gebiede und stede und auch furbas, so sie verriste mogen, und ane geverde geleide lihin gein der stat, da die wale geschehin sal, und hin wedirumb zo varen by der pene meynneidis und virluste siner stymme, die er zů der zyt an der kure habin sulte; und setzin, daz er adir sie in die buße zå stunt gefallin sin, die adir der an dem vorgenant geleide sumig sint adir daz geleide freveliche widdirsprechint.

[2] Darubir setzen wir und gebiedin andirn fursten gemeinlichin, wie sie genant sin, die gelehint sin von deme heiligen Romischen riche, und auch allin grefin landishern rittern knechten edel und unedel burgeren und gemeynen und allen insesses der burge und stede und allen steden des heiligen richis, daz sie zů der selbin zit, so man die kur dỉn sal eyns

${ }^{1}$ Kleidung (insigniis). - 2 Für die Worte „recht zů schuren“ keine Vorlage im Original; der ganze Satz ist in der Uebersetzung etwas umgestellt. - " "Dis ist geschehin" vorn Uebersetzer der Dentlichkeit wegen hinzugefügt. - Hs. genudit. 
Romischin konigis zů eyme kunfftigen keisir, eynen iegelichin kurfursten, so ers an sie forderd, adir sine boten, die er zů der kür sendit, sullen geleidin durch ir gebiede und so sie ferste mogen, alse vor bedacht ist, ane alle geverde. Wer auch die vorgeschrebin unsir gesetze frebeliche ubirginge, der sal zů stunt in die vorgenant buße gefallin sin: alle fursten und grefin landishern edel rittere knechte und alle wolegeborne, dye daweddir dunt, dye fallin in quot meyneydes und virlust allir leen, die sie von deme heiligen riche hant, und andir lehin, von weme sis hant, und als irs gutis, wo sie iz hant; alle burgir und gemeinde, die sich virmeßynt darweddir zů dunde, die sint gelich meyneydig und darzů sint sie allir ire recht friheit priviligia und gnadin, die sie von dem heiligen riche hant, gentzlich beraubit, und ir lip und ir gut fellit in keisirliche achte. Und ubir die selbin, die wir von nů dirre zit mit gedat als auch danne ${ }^{1}$ berauben von allim irrem rechtin, ist eyme yegelichin herleibit anzůgriffen ungefrebilt mit eygin gewalt ane gerichte unde anzůgriffin ${ }^{2}$ eins ieclichin amptmannis, und sullen darumb keine buße fochtin von deme riche adir von iemanne, umb daz sie begriffin werdin, daz sie weddir eyn gemeyne gut $^{3}$, uffinbere und des heiligin richis gesetze und wirdekeit und auch wedir eigin ere und heil also groß ding frefeliche gehindird hant und auch als wedirspenische ungehorsam ungetrewe alse boslich und ungetrewelichin hant gedan.

[3] Wir setzin und gebiedin, daz burger und gemeynne den vorgenant kurfursten, so sie iz von in fordern, schuldig sin zů virkeiffin und schaffin, daz man yn virkeiffe spise, so ir und iren boten und irs fulkys notdurfft, noch gemeyme lauffe und werd, so sie farn zi̊ der stat, da man die kur begen sal, unde auch, so sie von dannen farin; zů den vorgeschreben dingen sal niemans kein geverde legin; wer andirs darweddir dete, so wullen wir, daz er mit der gedede falle in die buße, die in dem vorgesetzen widdir burgir und gemeinne von uns uzgekundit sint. Welchir herubir von fursten grefin landisherren ritter $n$ burger $n$ und gemeynnen eyme kurfursten, so er ferit zů der kure eynen Romischen konig zů kiesin adir,

${ }^{1}$ ipso facto exnunc prout extunc. -2 sine iudicio sen invocatione.

$-{ }^{3}$ rempublicam, s. o. S. 109 A. 3. 
so er von dannen ferit, fintliche hude ${ }^{1}$ furmachit adir sich virmissit lage zi̊ legen adir sie adir etlicheme undir in an sinre adir sinre lude lip adir gut anegriffit, anebetrubit adir ir vorgenant badin ${ }^{2}$ (sie heischin geleide adir nit), den urteilen wir furfallin an der gedat in die vorgenant buße mit allin gesellin siner bosheit, also zů wise ${ }^{3}$, daz iecliche persone in die buße falle, die wir nach undirscheit der personen hievor han ufgesetzit.

[4] Wer auch, daz etzlichir kurfurste mit etwemme sime mitdekurfursten fintschafft druge und undir in wellichirleye krieg widdirsache adir missehellunge lieffe, die ding sullen in keine wis daweddir sin: eynre sy deme andirn gebunden zů geleidin und sine boten, die er zi̊ der selbin kure sendet, mit vorgesatztir wiße by der pene meynneidis und virluste sundir zů dem male siner stimme in der kure, alse for uzgesprochin ist.

[5] Wellichir auch andir furste grefe landishern ritter knecht edel adir unedel burgir adir gemeynne von den steden mit etlichem kurfursten adir mit me uzsir in widdir wurdigen willin dinge adir wilchirleie zweyunge krieg adir missehelle lieffe, doch so sullent die selbin dem selbin adir den selbin kurfursten adir yrin boten, die sie zů der selbin kure sendin, dar zů farin und her widdirumb zů komen geleide geben ane alle widdirrede und geverde, alse ir ieclichir sundirlich virmidin wulle die egenant buße, die von uns uf sie gesprochin sint; und wer darweddir dete, den urteylin wir zů stunt gefallin in die selbin buße. Zů eyner witern sichirheit und festekeit allir vorgesetz so heißin wir und wullin, daz alle kurfursten und andir fursten grefin landishern edele stede und ir gemeynne bestedegin mit briffin und mit yren eyden alle vorgesetze, und sullin sich darzi̊ virbinden mit guten trewin und ane argelist kreffteclichin zů fullinbrengen. Und wer sich hindirzůge die selben briffe zů gebin, der fellit mit der dat in die buße, die wir nach undirscheit der personen ieclichim besundir uffgelegit hant mit den vorgesetzen.

[6] Auch willich kurfurste [adir furste], wilchis ardis adir wirdekeit er ist, lehin heldit von deme heiligin riche, adir

1 hostiles custodias. $-{ }^{2}$ später meist: botin. $-{ }^{3}$ ita videlicet. 
grefe landishern adir edel adir der nachkomen adir irben die vor- adir nachgeschrebin unsir keisirlich gesetze und recht nit wullint haltin adir in frefeliche widdirsprechin, ist is, daz her ein kurfurste ist, zi̊ stunt so sullint die andirn sine medekurfursten den selben furbaz me $\mathrm{uz}$ ir geselleschafft sließin, und sal darbin [der] stymme der kure und der kurfursten stat wirdekeit und recht und sal auch nit ingesetzit werdin von der lehin wegin, die er von deme riche heldit. Abir ein andir furste adir ein edel man, als vor geseit ist, der da missedut an den selbin unsirn gesetzin, den sal auch glichirwiz nieman in lehin setzin, die er von dem heiligen riche adir von iemans andirs heldit; unde darzů fellit er zů stůnt in alle vorgesetzite buße, die sine persone anedreffint.

[7] Wie wir doch unvirscheidenlichin wullen und gesetzit han, daz alle fursten greffin landishern edele rittir ${ }^{1}$ knecht stede und ir gemeyne virbundin sin daz vorgesprochene geleide zu lihenne eyme iegelichin kurfursten adir sime boten, alse vor geredit ist, so han wir doch ir ieclichem die mynre ${ }^{2}$ nit sundirlich geleide und geleidis lude zůgeordenerit, die allirmeist darzů geschickit sint nach deme gelegir der gegin und stede, alz iz sich zů hant fullecliche ufinberit mit der nachgeschrebin rede.

[8] $\mathrm{Zu}$ dem irsten den konig von Beheim ein irtzschenke des heiligin richis sullin geleidin ertzebuschofe von Mentze, Babinbergir und Wirtzeburgir buschofe, der burggrefe von Nurenberg; die von Hohinloch, die von Wertheim, Brunecke und von Hanauwe; stede Nurenberg Rotenburg und Windisheim.

[9] Den irtzbuschoff von Kollin des heiligin richis irtzkantzelir durch Lamparten sullint geleidin irtzbischofe von Mentze und von Drere, der paltzgreve von dem Rine, der lantgreve von Hessin, die [grefen] von Katzinelinbogin, von Nassauwe, von Diestz; item von Ysenburg, von Westirburg, von Ronkel, von Limpburg und von Falkinstein; item stede Wetflar Geilinhusen Fredeberg.

[10] Den irtzbuschoff von Drere irtzkanzelir des heiligin richis durch Welschlant und in dem konigriche von Arlet sullint geleiden der irtzbischof von Mentze, der paltzgreve von Rine;

${ }^{1}$ Hds. richtir. - ${ }^{2}$ nichilominus.

Zeitschrift für Rechtsgeschichte. XVIII. Germ. Abth. 
andir werbe der von Spanheim, von Feldentz, von Nassauwe grefen, Ruwegreven, Wildegrefen, von Ysenburg, von Westirburg, von Ronkel, von Limpburg, von Dieschtz, von Katzinelinbogen, von Eppinstein, von Falkinstein; und die stat von Mentze.

[11] Den paltzgreven vom Rine ein drossesse des heiligen richis den sal geleidin der buschoff von Mentze.

[12] Den hirtzaugin von Sassin ein irtzemarschalg des heiligin richis sullin geleidin der konig von Beheim und die irtzebischofe von Mentze und von Medeburg und die bischofe von Babinberg und Wirtzeburg, der margreve von Missin und der lantgreve von Hessin; item die epte von Fulde und von Hirsfelde und der burgreve von Nurenberg; die von Hohinloch, von Wertheim, von Brunecke, von Hanauwe, von Falkinstein; und stede Erfurd Mulhusen Nurenberg Rotinburg und Windisheim. Den margreven von Brannenburg ein irtzkamerer des heiligin richis sullint geleidin alle, die zůnest genant sin, dye den hirtzaugin von Sassin geleidin.

[13] Wir wullin und setzin kuntlich, daz ein iegelich kurfurste, der solich geleide wil habin, also zitliche fordere, von den er iz gedenckit zů heischin, und den weg, den er farin wil, vor virkunde, daz die, wilche darzi̊ gesetzit sint, daz selbe geleide zů gebin, und also angemanit werdin, sich zitlichin und bequemelichen darzů mogen bereidin.

[14] Die vorgeschrebin gesetze, die von des geleidis wegin gemacht sint, herluchtin wir, daz man sie also sichirlichin sulle virsten, daz ein iegelichir, der vor genant ist adir villichte nit genant ist, wem iz heim gedihit, daz von eme daz geleide in solichir geschichte gefordirt wird, virbundin sin undir den bußen, die for begriffin sint, daz geleide zů gebin sundirlich durch sin lant und gebiede und auch furbaz, so er ferste mag, ane argelist.

[15] Andir werbe setzin wir und ordeneren, daz der irtzbischoff von Mentze, der dan zů ziten ist, sal iedem kurfursten besundir die kure virkunden mit sinen botin und mit sinen uffin brieffin; in den brieffin sal uzgeleit sin dag und zil, zusschin den die selbin brieffe zů den selbin fursten nach gemeyme geduncke komen mogin; die selbin brieffe sullint haldin, daz von dem dage in den brieffen uzgeleit innewendig 
dreyn den nesten manden sullin alle kurfursten und iegelichir besundir zů Frankinfurd uf den Meyn geinwurtig sin adir sullint iren guden glauphaftigen badin zů der selbin zyt und stat sendin mit irm fullin gantzen gewalde mit irin uffin brieffin mit ir iegelichis großem ingesegel besegelt, eynen Romischin konig zů kiesen zu fưrdirn in daz keisirdům. Wie auch und undir wilchir furme die brieffe sullin gemacht sin, und waz wirdekeit an in sulle unvirwendelich behaldin werden, und in waz wise. und mosse die kurfursten ir boten zů der kure sullin sendin und wie sie in den gewalt gebot und procuratoria ordenerin sullint, daz findet man geschrebin und clerlich uzgeleit an deme ende dis buchis ${ }^{1}$; und die selbe furme, die danne gebin wird, gebiedin wir und wullen von folkomen keisirlichem gewalt, daz sie also altzůmal gehalten werde.

[16] Wir heißin und setzin, so iz darzu komet, daz des keisirs adir des Romischin konigis dot kuntlich wird in deme bischtum von Mentze, daz der irtzbischoff von Mentze zů stunt innewendig eyns mandis frist, an einandir zů zelin von dem dage der kuntlichkeit des selbin dotis, kunt dů mit sinen uffin briffin ieclichim kurfursten sundirlich den dot und die virkundunge, alse vor geschreben ist; abe nů der selbe irtzbischoff an der endunge ${ }^{2}$ adir virkundunge sumig adir villichte laz were von der zyt darnach in dren manden, alse da abin zů nest in deme gesetze geordent ist, so sullin die selbin kurfursten von eygeme můde und ungerůffin umb die dogint irir trewe, von der sie schuldig sin daz heilige rich zů besorgin, zůsamenkomen [in] die vorgenant stat Frankinfurd [zu] kyesen eynen Romischin konig zů eyme kunftigen keisir.

[17] Iz sal auch ein ieclich kurfurste adir sine botin in der zyt der vorgesprochin kür faren in die egenant stat Frankinfurd nit me dan myt zweinhundirt pherdin geredin, in der zal mag er mit yme furin funfftzig gewapinde adir minre und nit me.

[18] Sundir ${ }^{3}$ der kurfurste, der zů der kure geruffin were und zů der kure nit queme adir sine rechtliche boten mit

1 d. h. der Nürnberger Beschlũsse; unten Cap. 19. - ${ }^{2}$ executio. ${ }^{3}$ vero 
sinen uffin brieffin mit sime großim ingesegel besegelt, die follin frien gantzin gewalt hiltin eynen Romischin konig zů kiesen, nit sente adir, ob er queme adir die selbin boden villichte sente, und darnach der furste adir sine botin inweg fure von der kure, und ein Romischer konig zů eyme kunfftigen keisir noch nit gewelit were, und auch nit ließe noch insetzte wirdeclichin eynen waren procurator zu den vorgenant dingen, der sal darbin siner stimme und recht, die er zů der kure zo̊ der selbin zit hatte und sie also gelaßin hat.

[19] Wir setzin auch den burgern von Frankinfurd uf und gebiedin in by der crafft des eydes, den wir in darubir uffsetzin zio sweren zů den heiligen, daz sie mit getrueme ernste und sorgsame fliße behudin und beschirmen alle kurfursten und ir iegelichen von ubirfalle des andirn, ob it weddirwintigest undir in uffstunde, und auch von allen luden mit allem irem fulke, die sie und ir iegelichir in der vorgenant zal der zweir hundird pherde in die stat zů Frankinfurd gefurd han; andirs sie fallint in missetat meyneidis und virliesin auch gentzlich alle ir recht friheit wirdekeit gnade und laube, die sie von deme heiligen riche hant; und mit der dat fallen sie in keisirliche achte mit libe und mit gůde; und sy von stunt herleibit eyme iegelichen anzuggriffin ungefrevelt mit eygen willen und gewalte ane gerichte die selbin burgir, die wir umb die geschichte von dirre zit alse auch darnach beraubin alle ir recht alse virredir ungetrewin und des richis weddirspenschin; also wer die selbin angriffit, der sal in keine wise fochtin keinirleie buße von deme beiligen riche.

[20] Darubir die burgir von Frankinfurd durch alle die $z y t$, in der sich geburit ubir die kure zů dedingen, so sullen sie in keyne wis nyeman in die stat laßin, willichirleie wirdekeit adels adir hirschefte er ist, alleine die kurfursten uzgenomen und ir boten und ir procurator, der ir iegelichir mit zwein hundird perdin sal infaren, alse for gesetzit ist. Were abir, daz nach der kurfursten infard adir in ir gegenwurtekeit sich geburte, daz ieman in der stat funden wurde, des izfard sullin sie zi̊ stunt mit den werkin ${ }^{1}$ bestellin ane allis virziehin undir allen den bußen, dye dafor ubir sie herzalt sin, und auch in

1 cum effectu 
der crafft des eides, den die burgir darubir sweren sullin zů den heiligen by der crafft dys geinwurtigen gesetzis, alse dofor uzgeleit ist.

\section{Das andir capitel.}

Von der kir eyns Romischen konigis.

[1] Darnach so die kurfursten sint komen in dye stat zů Frankinfurd, des nesten morgens, so der dag uffget, sullin sie in ir allir geginwurtekeit in santte Bartholomeus kirchin daselbis dun singin eyne messe von deme heiligen geiste, daz er yn ir hertze irluchte und daz liecht siner crafft in ir sinne gisse, daz sie mit syner helfe gesturit werdin eynen gerechtin gudin und nůtzin menschin kiesin einen Romischin konig zů eyme konfftigem keisir und zi heile allin cristin ludin. Do die messe gedan ist, so sullin die kurfursten alle zi deme altir gen, uf dem dye messe gesungen ist: alda sullint die geistlichin fursten ubir dem ewangelio sancti Johannis ,in principio erat verbum etc.", daz daselbis fur sie sal geleit werdin, ir hende mit wirdekeit legen uf ir brust; die werntlichin fursten sullint mit irin hendin liplichin daran griffen, die alle mit allem irim gesinde sullin da geinwurtig sten ungewapint. Und der irtzebuschoff von Mentze sal in die furme des eidis gebin und er mit yn und sie mit yme adir der bodin, die nit geinwurtig sin, den eit in Dutschin do̊n in dese wise:

[2] „Ich irtzbischof von Mentze des heiligin richis irtzkantzelir durch Dutschelant und ein kurfurste sweren zů desin heiligen ewangeligen, die hie geinwurteclichin fur mir ligin, daz ich uff die druwe, mit der ich gode und deme heiligen Romischin riche virbundin bin, kiesin nach allir miner bescheidinheit und virnufft und mit godes helfe kiesin wil ein zitlich houbit cristim folke, (daz ist ein Romisch konig zů eyme kunfftigen keysir, der darzů bequemeliche sy,) alse verre mich mine bescheidinheit und mine sinne wisint, und by der selbin trewe; so wil ich gebin mine stimme und willin und die vorgenant kůre di̊n ane alle gedinge miede lon adir gelobede, adir wilchir wise soliche ding mogen genant werden. Alse helfen mir got und alle heiligen."

[3] Do der eit von den kurfursten adir irin boten in der vorgenant furme und wise gesworin ist, so sullin sie sich 
schicken zů der kure und sullint von der stunt uz der stat zů Frankinfurd nummer von einandir komen, iz inhabe dan daz merer deil undir in vor gewelit eyn zitlich houbit ein Romischin konig cristem fulke zu eim kunfftigem keisir; wirzogin sie daz zů dunde drißig dage nach einandir zů zelin von dage des gesworin eidis, darnach daz die drißig dage uzsint, sullint sie furbaz me nit eßin dan waßir und brot und sullint in keine wis $u z$ der stat nummer komen, ez sy dan for von in adir von dem meisten deile undir in ein zitlich houbit der heiligin cristenheit irkorn.

[4] Nachdeme daz sie adir der merer deil undir in gewelit han, die selbe wal sal gehabit sin und behaltin werdin, alse ob sie eynmudeclichin von in allen were geschehin; geschehe iz auch, ob etliche kurfursten adir ire boten durch etliche zit nit geinwurtig werin und sich virspedet adir gehindird wurdin und doch quemen, e die vorgenant kur volendit wurde, dem bekennen wir, daz man in zi̊ der kure laßin sal in dem selbin wesin, in dem sie ist in der zyt siner zůkunfft. Wan $^{1}$ is nů von altir bewertir und lobelichir gewonheit ungebrechinlichin bisher gehaltin ist, daz hienach geschrebin stet, darumb so setzin wir und bekennen von fullenkomen keisirlichin gewalt, daz der, der in forgesatzte wise irkorn wird, zů eime Romischen konige, so die kure geschehin ist, e er sich keinirleie sache adir gescheffte von krafft des heiligen richis it undirste zů důne, so sal er allen unde bisundir den kurfursten geistlich und werntlich, von den kuntliche ist, daz sie die nestin gledir sint des heiligin richis, alle ir privileige briefe recht friheit furluhin ding alte gewonheit und wirdekeit, und waz sie von deme riche bis an den dag der küre gehabit han und besessin, bestedegin und befestin mit sinen besegeltin briefen ane furzog und weddirrede; und die vorgenant stucke sal er in alle hernuwin, nach deme daz er mit keisirlichir imfeln gekronit wird. Die bestedunge sol der herkorn eyme iegelichim kurfursten bisundir důn von irst mit sime koniglicheme namen und darnach undir siner keisirlichin wirdekeit hernuwin; in den dingin sal er dye furstin alle in der gemeyne und ir ieclichin bisundir nummer gehindirn, me er ist sie schuldig gnedeclichin zů furdern ane argelist.

${ }^{1}$ Hds. von; s. auch S. 109 A. 8. 
[5] In der geschichte, so dry kurfursten geinwurtig adir der boten, die nit geinwurtig sin, den firden undir in adir uz ir geselleschaffte, daz ist ein kurfurste, der geinwurtig adir nit geinwurtig were, zů eyme Romischin konige irwelten, deme irkennen wir mit gesetze, daz des irwelten stimme, abe er geinwurtig were adir sine boten, ob yme nit geburte geginwurtig zi̊ sine, volle crafft habe und die zal der kore mere und daz groBir deil hersetze glich den andirn kurfursten.

Daz dritte capitel. Von deme sitzin der irtzbischoff von Mentze Dryere unde Ko̊llen.

In namen der heiligen undeilsamen drivaltekeit seliclich. Karlus der firde von gunste gotlichir gnade alle zit merer und konig zi̊ Beheim. Zi̊ ewigem gehugniße ${ }^{1}$ der dinge. Gezirde und ire des heiligen Romischen richis und keisirlichir wirdekeit und dangbir nuttz gemeynes gudis furet einmudegir wille der wirdegin herluchtin kurfursten, die alse hohe sule fursichteclichir wisheit den heiligin bů mit sorgsamyr miltekeit [ufrecht haltint], mit der helfe keisirlichis gewaltis recht ${ }^{2}$ wird gestirkit, und so sie me mit witer mynne gelichis gunstis mit ein virstrickit sin, so sie me seleclichin uzgißin gemach fruchtberis freden und ruwe allir cristenheit. Darumb daz undir den wirdegin irtzbischofin von Mentze Kollin und Drere des heiligin richis kurfursten allir krieg und argwan, die undir in mochtin ufsten von deme furgange und umb wirdekeit irs sitzins in keisirlicheme adir in koniglicheme hofe, hienach und furbas me zu ewigin ziten abesin und sie in geruwigir wise hertzin und gemudis blibinde ${ }^{3}$ quemelichir bedrachten mogin mit gemeime gunste und lißße dogintsamer liebe die notdurfft des heiligen richis zů droste cristim fulke, so setzin wir mit vorbedrachtunge mit allin kurfursten geistlich und werntlich und mit irme rade unde von fullenlsomenheit keisirlichis gewaltis bestedegin wir mit desem gesetze ewecliche $\mathrm{zu}$ werin, daz ${ }^{*}$ die irtzbischofe mogin und sullen sitzen in allin uffinlichin keisirlichin und koniglichin gededen, an gerichte, lehin zo̊ lihin, $z u$ dissche eBin, in reden und in

${ }^{1}$ memoria $-{ }^{2}$ quorum presidio dextra imperialis potentie roboratur $-{ }^{3}$ Hds. blibin die $-{ }^{4}$ Umstellungen gegenüber dem Original. 
allin andirn werckin, adir so in geburit zůsamene komen zů dedingen umb des richis nůtz und ere: der bischoff von Drere sal gelich geyn des keisirs antzlitze sitzin; der von Mentze sal in sime bischtum und in siner profincien und auch uzwendic siner provincien in allir sinre Dutschen kantzelarien ane in der provincien von Kollin sitzin zů der rechtin siten des keisirs; der von Kollin sal in sime bischtum und in siner provincien und uzwendic siner provincien in allem Lamparten und Welschlant sitzin zů der rechtin hant des keisirs. Wir wullen, daz die selbe wise zů sitzin by allir ordenunge, alse vor gesprochin ist, gehaldin werde von den nachkomelingin der vorgenant irtzbischoffin Kollin Drere und Mentze, daz zu keiner zyt umer me keinerleie zwifil von den dingen ufsten.

Das IV. capitel. Von ${ }^{1}$ deme sitzin allir kurfursten und von dem zůruffin der kurfursten eynen Romischen konig zư kiesin und von den amptin der werntlichen kurfursten keisirlichis hofis et cetera.

[1] Hienach setzin wir, wie dicke iz sich heischit von desir stunde und furbaz me, so ein keisirlichir hoff gehaltin wirt, (in iegelichim sitzen zů rade zů dyssche und in wilchin andirn stedin, da eyme keisir adir eim Romischin konige mit den kurfursten geburit zi̊ sitzin,) so sal der konig von Beheim, umb daz er kronit und gesalbit furste ist, allirneste sitzin by dem irtzbischoff, er sy von Mentze adir von Kollin, dem dan zů zidin ane mittel geburit zů sitzin by der rechtin sitin des keisirs nach geleginheit der stede und der provincien, alse ir friheit heldit; nach deme konige von Beheim uf die selben siten sal sitzin der paltzgreve von Rine; abir zů dem linketen deil nach dem vorgenant irtzbischof, [dem zu dem linketen deil] ane mittel geburit zu sitzin, sal der hirtzauge von Sassin sitzin, darnach der margreve von Brannenburg.

[2] Andir werbe, wie dicke und wanne daz heilige rich ledig ist, von dannen sal der irtzbischof von Mentze gewalt han, (als iz auch bekant ist, daz er von altir her hat gewalt gehabit die andirn kurfursten, sine gesellin in der kur, mit brieffin by ein heischin, die alle adir die geinwurtig mogen

1 Im Or. nur: De principibus electoribus in communi. 
adir wollin sin) und in dem zil der kurfursten by ein gesament, so sal der bischoff von Mentze und kein andir stymme innemen undir solichir ordenunge: ziom irstin so sal der irtzbischoff von Mentze den irtzbischoff von Drere anfragin, dem die irste stimme zugehorit, alse iz von altir herkomen ist; darnach den irtzbischoff von Kollin, dem die wirdekeit und daz ampit zůgehorit, daz er eym Romischin konige die irste konigliche krone uffsetzit; zum drittin male von deme konige von Beheim, der undir leyenschen furstin von koniglichir wirdekeit den anefang heldit; zum firdin male von deme paltzgreven von Rine, zum funfftin male von deme hirtzaugen von Sassen, zum sestin male von dem margreven von Brannenburg. So daz allis geschebin ist, so sullint die vorgenant furstin den bischoff von Mentze irin gesellin wedirumb fragin, daz er in sine meinunge und sinen willen ufinbare. Andir werbe in der hirschafft eins keisirlichin hofis sol der margreve von Brannenburg waßir gebin ubir des keisirs hende adir des Romischin konigis; den drang sal dienen der konig von Behem, daz er doch nit schuldig ist zü dunde undir siner koniglichir kronen, er wulle iz dan gerne dun von willin; der paltzgreve von dem Rine sal daz irste eßin anedragen, der hirtzauge von Sassin marschalg sal sin ampt dun, als is von altir her gewonlich ist.

\section{Das V. capitel.}

Von des paltzgreven und des hirtzaugin von Sassin ampten, so daz beilige rich nit houbitis hait etc.

[1] Wye dicke iz hienach geschicht, daz daz heilige rich ledig stet, so sal der paltzgrafe von dem Rine furmundir sin des heiligin richis von der paltzgreveschafft wegin zů der hant eins kunftigin Romischin konigis in den landin des Rines und Swabin und in Frenschin gebiede mit gewalt, gerichte zů haltin, geistliche gabe zu lihin, gulte und gefelle insamen, in lehin zu setzin, eide umb getruwekeit an des heiligin richis stat und in des selbin richis namen zů inphahin; daz selbe sal doch allis zů siner zit von deme Romischen konige, der darnach herwelit wird, hernuwit werdin; und sal man yme die eide alle von nuwem uffswerin; alleine uzgenomen der furstin lehen und vanlehin, der lyhin und insetzin behaltin wir be- 
sundir alleine dem keisir adir eyme Romischen konige. Doch sal der selbe paltzgreve wißin, daz yme uffinlichin virbodin ist alle virandiringe adir virsetzin des richis gut, die zit er furmundir ist. Der hirtzauge von Sassin sal ein furmundir sin an allin steden, da man Sassin-recht heltit, undir allir wiße und maße, alse dafor uz ist geleit.

[2] Und wie doch iz sy von altir gewonheit ynbrocht, als man sprichit, daz ein keisir adir ein Romischir konig in sachin, umb die er hat zi̊ antwurten vor eynem paltzgraven des Rines, der da ist des heiligin richis ein irtzdrossesse und ein kurfurste, doch so mag der selbe paltzgrafe daz gerichte nirgin andirswo gehaltin dan alleyne in des richis hoff, da der keisir adir der Romische konig gegenwurtig ist.

\section{Daz VI. capitel.}

Von der kurfursten wirdekeit an sitzin an gen und an sten gein andirn gemeynen fursten ${ }^{\mathbf{1}}$.

Wir setzin ewecliche, wie dicke iz sich furbaz me geburit, daz ein keisirlich hof gehaltin wird, daz die vorgenant kurfursten geistlichin und werntlichin nach der vorgeschrebin ordenunge und wise ir stede zů der rechtin und linketen hant unvirwandelt haltin, und sal in adir yr keyme in wilchirleie gededin, die in den hof horint, kein andir furste, waz wirdekeit und ardis er sy, in kein wis furgezagin werden an gen, an sitzin adir an sten und bysundir, daz mit namen der konig von Beheim in der hirschafft solichis hofis en allin und sundir vorgenant stedin und dedin unvirwandellich sal furgen eyme iegelichen andirn konige, in waz friheit adir wirdekeit er herschinet, der ${ }^{2}$ villichte von etzlichir geschichte adir sache gedege darzů komen adir do sin.

Daz VII. capitel. Von der fursten nachkomen.

[1] Undir den unzellichin sorgin, von den unsir hertze degelich virmudit wird, umb daz selige wesin des heiligin richis, daz wir von gote merer ${ }^{3}$ seleclich besitzin, so ist unsir bedrachtin zůfur darzu berichtit, wie die begirliche heilsům

1 Im Or.: De comparatione principum electorum ad alios principes communes. $-{ }^{2}$ Hds. dem. $-{ }^{3}$ deo auctore. 
eynnekeit alle zyt undir den kurfursten des heiligin richis stedecliche grủne und ir hertzin in eynekeit lutir minne inthaltin werdin, von deren fursichtekeit der wildin wernt so snellir so lutirlichir zů rechtir zyt geholffin, und so vil undir den kurfursten kein wersal sich virzuckit und minne lutirlichin wird behut, und virborgin krot ist abegesnedin, so iegelichis recht luthirlichir wird irluchtit. Wan nư gemeinlich lang und breit uffin ist und villichte durch alle die wernit kuntlich, daz die hirluchtin konig von Beheim, paltzgreve des Rines, hirtzauge von Sassin, margreve von Brannenburg von des richis crafft und irre furstendum in der kur eins Romischin konigis zi̊kunftigen keisirs mit den andirn geistlichin kurfursten rechte stimme und stat habin und eyne mit den andirn gesetzit sint, daz sie ware und riliche ${ }^{1}$ kurfursten sint des heiligin richis. $\mathrm{Daz}^{2}$ nů it in kunftigen ziten materie ergirůnge und missehelle uffsten undir der kurfursten sone adir ir nachkomen von recht stimme adir gewalt zi der kur und alse ein gemeyne gut mit kunftigem schedelichin virziehin wurde gehindird, dem schadin zů begenen heilsameclich mit gotz helfe, so setzin wir und bekennen in keisirlichem gewalt mit desem geinwurtigen gesetze ewecliche zů blibin, nachdeme daz dye selbin kurfursten und ir iegelichir dot ist, des recht stimme und crafft zů kiesin sal fallin an sinen irsten elichin geborin son, der ein leie ist; ob der nit were, so sal die kure frilichin fallin uf des selbin irstin geborin son elich und leie ane weddirrede eins iegelichen. Were abir, daz der selbe irste geborn son von dirre wernde vor virfüre ane knabin-irbin eliche leie, so sal von crafft dis keisirlichis gesetzis recht stimme und gewalt der kure fallin uf den altirn brudir leie, der von warem fedirlichim stamme ist komen, und danach uff des selbin irstin geborn sone; soliche nachkomen ${ }^{3}$ sal ewecliche behaltin werden an den irsten geborin sonen und irbin der kurfursten an recht stimme und gewalt vorgenant, doch also ob ein kurfurste adir sin neste geborn son adir andir sine sone leie sturbe, und gedege, daz er ließe knabin-irbin elich leien, die nit follen alt werin, so sal der altste brudir des dodin des selbin dodin kindir furmundir sin und besorgen, biz daz altiste undir den

1 legitimos. $-{ }^{2}$ Construction! $-{ }^{3}$ successio. 
kindern zů fullim aldir komit; (daz altir acbtin wir an eym kurfursten achtzehin jar fullenkomen, und wullin und setzen, daz iz ewecliche alse gehaltin werde;) und wanne der altir son zů dem altir komit, so ist der furmundir zů stunt schuldig umb in zu geben recht stimme und gewalt mit ampt ${ }^{1}$ und allis, daz darzů gehorit.

[2] Were iz auch, daz etlichiz des selbin furstendum dem riche ledig wurde, von deme sal der keisir adir Romisch konig, der dan zu den ziden ist, fursehen als von dingen, daz an in und an daz riche mit rechte gefallin ist; alle zit unsirm konigriche von Beheim, so iz ledig ist, behaltin [wir] sine friheit recht und gewonheit ubir die kur eins konigis von Beheim, die geschehin sal von den inwonendin des richis, alse ir privileigia haltin und von langir gewonheit gehaltin hant von kristenlichin keisirn und Romischin konigin: den fribeit rechtin und gewonheit wullin wir mit desen selbin keisirlichin gesetzen in keyne wis schadebir sin unde nach me wir setzen und virendungin nů und zů ewegin kunftigen ziten, daz die selbin friheit recht und gewonheit mit allir ir schrift und furme unzwivellichin mit festekeit irre crafft gehaldin werdin.

\section{Daz VIII. capitel.}

Von der friheit des konigis von Beheim unde siner lute.

Alse iz hievor den cristlichin konigin von Beheim unsirn altirn und furfarn und dem konigriche von Beheim und des selbin richis krone gnedeclichin ist virluhin und virhengit von den Romischin keisirn und konigen unsirn furfarn und nach in dem selbin riche von der zit, dawedir hut dis dagis kein gedenckin ist, von labelichir forbeschrebener gewonheit von langin ziten und mit gewonheit der gebruchinden inbracht ist ane wedirrede und ane alle wedirwurdekeit gehaltin ist, daz kein furste landisherre edel rittir knecht burgman burgir, nachdan ${ }^{2}$ kein persone des selbin richis und allis, daz darzu horit, an welchin enden daz ist, zu keins clagirs ansprache, was wesins wirdekeit adir ardiz der selbe clagir ist, inmoge noch insulle geladin gezogin noch geruffin werdin an keinerleie und yemans andirs gerichte dan alleine vor des konigis von Beheim und des koniglichin hofis richtir adir gerichte: herumb

${ }^{1}$ Hds. ampmit. $-{ }^{2}$ denique. 
so irnuwin wir und bestedegin wir von sichirm wißin und setzin mit unserm geinwurtigem keisirlichem gesetze mit fullinkomen keisirlichim gewalt die selbin friheit gewonheit und gnade eweclichin stede zů haldene, ob ieman von den vorgenant, er sy furste landisherre edel rittir knecht burgir burgman adir gebure adir andir willichirleye vorgenant persone zů iemans gerichte uzwendig des konigrichis von Beheim geladen wurde wedir die vorgenant friheit gewonheit adir gnade in lastirbere adir schultbere adir virmissthetin sachin adir in welchen andirn geschefftin, zů wilchir zit daz geschehe: die persone, die also geladin wurde, ist nit schuldig zü irschinen adir zů antwurten an gerichte andirs, dan vor geschrebin stet. Ob ni̊ von eim richtir, von weme der gesetzit were adir was gewaltis er were, uzwendig des konigrichis von Beheim wedir den selbin, der nit vor gerichte irschene, in gerichtis wise gefarn wurde mit urteile, die urteil sie sin undirsprochin adir geendit urteil, eyne adir me, in wilchin sachin adir gescheften daz geschehe adir in waz wise sich geburte, daz die selbin urteil uzgegebin und gekundit wurdin: die ladůnge gebot processe und urteil und allis, daz von in in gerichtis wise mage geschehin adir virsucht werdin, daz virnichtin wir allis und duns gentzlichin abe von fullemkomenheit keisirlichis gewaltis. Darzů legin wir uffinlichin und setzin mit desem keisirlichem gebode vorgenant gewaltis ewecliche zo̊ werinde, als iz in dem vorgenant riche zi Beheim fließeclichin gehaltin ist von der zyt, dawedir nieman kein gedencken hat, daz ubiral kein furste landisherre edel rittir knecht burgir burgman gebure nach kein persone adir inwonende des konigrichis von Beheim, willichirleie wesins wirdekeit adir ardis er sy, sullint sich nummyr beruffen von keime gerichte urteil adir geboden des konigis von Beheim adir sinre richtir unde sullin sich auch nummer beruffin zi̊ eyme andirn gerichte, waz wedir sie an koniglichem gerichte adir des richis richtir gedan adir gesprochin adir gehaltin ist adir hienoch wiedir sie gesicht adir gesprochin wird. Geschicht iz abir, daz etliche beruffunge an gerichte adir uzwendic gerichte geschehen, zu stunt sullin die selbin beruffin mit keynir craffit besten, und die sich beruffin han, sullint wißin, daz sie zů stunt fallin in buße virlustekeit irre sache. 


\section{Das IX. capitel.}

Von den gultgrubin in dem riche zů Beheim ${ }^{1}$.

Wir setzin mit desem geinwurtigem gesetze ewecliche zů werinde, daz unsir nachkomen konige zů Beheim und alle kurfursten geistlich und werntlich mogint han gultgrůbin und andir grubin von smedesamen dingin und saltzgrubin, die itzunt fundin sint adir hernach fundin werdin, in deme Beheymschen rich adir in landin, die deme selbin riche undirdenig sin mit allin rechtin, alse sie iz bizher gewonliche besessin hant. Sie mogen auch Judin han und zulle nemen, alse iz hievor uffgesetzit ist und mit lobelichir bewertir gewonheit von langin ziten an unsere seligin altirn konige zů Beheim und an die kurfursten und ir altirn mit virschrebeme lauffe rechtecliche bekant ist zů behaltin.

Das $X$. capitel sagit von den muntzin und andirn gnadin des richis zů Beheim et cetera ${ }^{2}$.

[1] Wir setzin furbaz me, daz die konige zů Beheim in irme riche und in allin landin, die deme riche zůgehorin, an welchin steden sie wullint, mogint dun muntzin slahin von gulde und silbir undir allir wise und furme, als iz in deme riche zu Beheim uff dise zit in fredesamir stedir besitzunge gehalten ist. [2] Auch wullin wir mit desim keysirlichem gesetze und unsir gnade ewecliche zů werinde, daz den kurfursten konigen zů Beheim herleibit sy von eyme iegelichim fursten hern grefin und andirn luden ein iegelich lant burge irbe eygin adir gut zů keiffin und an sich zů gewinnen, in gabe adir in phandis wise uffzunemen undir gewonlichem gedulde der lande burge irbe eigin adir gut, also zů emphahin adir keifin eigin fur eygin, lehin vur lehin, also daz die konige zů Beheim von den selbin landin burge irbe eigin und gut deme heiligin Romischin riche virbundin sin zů gebin und zů dienen sin gewonlich recht. [3] Dis geinwurtige gesetze und gnade streckin wir mit crafft unsirs keisirlichin rechtis zů allin kurfursten geistlich und werntlich und zů iren nachkomen und ir elichen irbin mit allir wise und gedinge, alse vor geschrebin ist.

${ }^{1}$ Sic! Or. De auri argenti et aliarum specierum mineris. - ${ }^{2}$ Sic! Or. De monetis. 


\section{Das XI. capitel.}

Von friheit der kurfursten des Romischin richis.

[1] Wir setzin auch, alse wir fundin han, daz in alten gezidin gehaltin ist, daz keine grefin landisherrin edel gelehinten dinstman burglude rittir knecht burgir und burgsessen adir kein persone, in wilchim wesins ardis adir wirdekeit die undirdenig sind den stifften Kollin Mentze und Drere, nit mogint geladen noch beclagin noch bezagin adir geheischen werden zi̊ iemans ansprache an keim gerichte uzwendig gebiede der stifte dan alleine fur gerichte der irtzbischof und irin richtirn, und also daz bizher gewesin ist, also sal iz fur wirtir ewecliche gehalten sin. Were iz abir, daz die undirdan der vorgenant stiffte weddir unsir dis gebot zů iemans ansproche wurden geladin an iemans gerichte uzwendig gebiede und gemircke der vorgenant stiffte, in waz sachin das were, umb ere adir umb gut adir gemissthete sache, der ist nit schuldeg zi irschinen adir den gerichtin zu antwurten; und die selbe ladunge und processe unde alle urteil, wie man sie nennit, die weddir die nit komendin geschehin adir gesprochen werdin von den uzsirn ${ }^{1}$ richtirn, und allis, daz daruz gelaufin mag, herkennen wir fur nicht mit dem, so iz geschicht. Darzů legin wir und setzin, daz grefin landisherrin edel gelehinte dinstmanne burgmanne rittir knecht burgir gebure nach keinerleie persone, die den selbin stifftin undirdenig sint, wilchirhande wesins wirdekeit adir ardis sie sint, der keime ist herleibit sich zi beruffen von den gerichtis wise urteil adir geboten, wie sie genant sint, die wedir sie gesprochen sint adir gesprochin werdin an der irtzbischofe adir irre zitlichin amptlude gerichte, aldiewile daz in an den selbin gerichtin rechtis nit virsagit wird, von den sie claginde sint; waz beruffunge hiewedir geschehe, die sal man nit inphahin, und kundin [wir] sie virnicht; abir an gebrestin der gerechtekeit so sy allin vorgenant herleibit, in den sachin yn recht virsagit wird, sich zů beruffin unde alleine zu keisirlichem hofe und gerichte adir vor des richtirs gehorde, der zu der zit gerichte besetzit in keisirlichim hofe, und fur keinen andirn richtir; waz hiewiddir geschehe, daz sy zi̊ stunt virnichtit. [2] Daz selbe gesetze

1 a iudiciis extraneis. 
wullin wir mit crafft unsirs geinwurtigen keisirlichis gerichtis geschickit han zů den herluchtetin werntlichin kurfursten paltzgrave hirtzauge von Sassin und margrafe von Brannenburg und zu irin irbin nachkomen und undirdan fulleclichin undir allir wise und gedinge, also vor gesagit ist ${ }^{1}$.

Das XII. capitel. Von samenunge der kůrfursten.

Undir den manigfeltigen sorgin eins gemeynnis gutis, in den unsir gedang dicke inzuckit wird, so hat unsir wirdekeit angesehin, daz iz notdurftig sy, daz die kurfursten des heiligin richis, die da sint feste stutzil und unbewegeliche sule des richis, me züsamene komen, dan bizher gewonheit ist gewesin, zů sprachin und zi̊ dedingen von des richis und der wernde heil, alsie durch ferre geleginheit der lande einre von dem andirn wonende sint, so vil sie baz konen gesagen und mit einandir geredin von den inbrechinden gebresten der lande, die in bekant sin, und kunnent mit gesundim rade irre fursichtekeit heilsame helfe gebin und gemachesame widirbrengen solichin gebrestin. Hievon ist, daz in dem hohin unserm hofe ${ }^{2}$ zu Nurenberg, der durch unsir wirdekeit wirdeclichin begangen ist mit den irlichin geistlichin und herluchtin werntlichin kurfursten und mit andirn furstin und herrin, da han wir mit bedrachtim muide mit den selbin kurfursten und mit irim rade geordenerit umb ein gemeynne gut und heil, daz die selbin kurfursten furbaz me zi̊ eim male aller jar vier wochin nach dem ostirlichin dage selbir sullint zůsamenkomen in etzlichir stat des richis; und nů zu der selbin zit dis geinwurtigen jaris sal von uns und den selbin furstin begangen werdin gespreche und hoff mit der selbin samenunge in unsir keisirlichir stat zu Metze, und da sal von uns mit der furstin rade bescheden werdin stat der vorgenant samenunge, welchis dagis furbas me in dem nachgendem jare man zusamene sulle komen; dese ordenunge sal alleine werin zu unsirm und der furstin willen. Diewile die ordenunge werit, so nemen wir die kurfursten in unsir keisirlich geleit zi̊ deme vorgenant hofe ${ }^{2}$ zů komen zů blibin und auch wedir heim zu varin. Herumb

1 Die kaiserliche Interpretation dieses Capitels, welche in dem Böhmischen Exemplar der G. B. zwischen 1366 und 1378 in margine zugefügt worden ist, fehlt in dieser alten Uebersetzung. $-{ }^{2} \mathrm{Hds}$. hafe. 
daz daz gespreche und rat gemeines gudis und fredin nit gehindird wurdin mit virziehin adir blibin in droste adir mit ubirmeßigeme fliße der wirtschafft ${ }^{1}$, alse iz etwan gewonlich ist geschehin, so ordenerin wir mit gemeymme willin, daz furbaz me, diewile der vorgenant keisirliche hoff adir samenunge werd, keim furstin irleibit ist gemeynne sproche ${ }^{2} \mathrm{zu}$ haltene; abir sundirlich gespreche, $\mathrm{daz}^{3}$ die ordenunge der keisirlichin dingen, die zu dunde sint, nit irrint, die sint mit maßen virhenget 4 .

Daz XIII. capitel. Von deme wedirruffen der friheit.

Andir werbe setzin wir und bestedegin mit desem keisirlichim gebode, daz alle friheit und briefe, welchin personen, was wesins furnemekeit adir wirdekeit sie sint, adir stedin frien adir eygin, die von uns adir von unsirn seligin furfarn gebin sint, undir wilchim inthalteniße der worte ubir recht gnade friheit gewonheit adir gut, die von uns adir von unsirn seligin furfarn Romischin keisirn und konigen von eigin willin adir yn andirs virluhin sin adir hienach von uns und von unsirn nochkomen Romischin keisirn und konigen virlubin wurden, die insullint noch inmogint gentzlich an keinen dingin geletzin adir abegezihen den friheidin gerichtin rechtin eren adir hirschefftin der kurfursten geistlichir und werntlichir; were iz auch, daz in solichin friheiden und briefin etlichir personen, waz furnemekeit adir wirdekeit sie werin adir gemeyne, alse e genant ist, uzgenomelichin besorgit were adir hienach virhut wurde, nit sullint sie wedirruffinlichin sin, ez inwere dan, daz von den selbin brieffin und fribeit undir allin worten inen begriffin ${ }^{5}$ nach einandir sundirlich rede geschehe von wortin zů wortin. Die selbin brieffe und friheit, nach dem und also vil alse sie abeziehinde sint den friheiten gerichten rechtin eren adir hirschafft der vorgenant kurfurstin adir ir ieglichim an deme ende, wedirruffin wir sie von sichirme wißin und dun sie abe und setzin von keisirlichim gewalte, daz man sie virste und halte, daz sie wedirruffen sin.

${ }^{1}$ frequentationem convivii. - ${ }^{2}$ generales invitatas. $-{ }^{3}$ sic! 4 permisse. - ${ }^{5}$ Hds. dahinter: wurde! 


\section{Daz XIV. capitel.}

Von den, die erin eygen herren boslich wedirsagen ${ }^{1}$. In etwie vil landin iz geschicht, daz dinstman und gelehinte manne die lehen und die gulte, die sie von den selbin herrin hant, mit wortin und mit geverden uffsagin untzitlich und uffgebint; und so die selbe uffgabe geschehin ist, so weddirsagin sie boslich den selbin herrin und dunt in ir fintschafft kunt; und darnach dunt sie in großin schaden und die selbe gulte und lehin, die sie von der fintschafft und kriegis wegin hant uffgegebin, die griffin sie an und bekomern und haltin sie. Herumb setzin wir mit desem geinwurtigem gesetze ewecliche zu werinde, daz die selbe uffgabe sal man virnicht han, als ob sie nie geschehin were, iz insy danne, daz die selbe uffgabe und uffsege von in frilich und mit werkin ${ }^{2}$ geschehe, also daz daz selbe gut gulte und lehin den herrin von yn liplichin und redelich werde ${ }^{3}$ uffgebin in solichir wise, daz die selbin wedirsagere die herren an den selbin guten und gulte und lehin nummer zi keiner zyt betrubin adir schedigin von yn adir mit andirn, noch insullint darzů rat helffe adir gunst nummer gebin". Wer darweddir dut adir die herrin an den selbin gutin lehin adir an andirn irren guten betrubit adir in schadin brengit adir wer dem, der soliche ding dut, rat und helfe irbudit, der virlusit zü stunt die selbin lehin und gulte und ist irin loiz und fellit in des richis achte, und sullin furbaz me zů allir zit kein weg uffin sin noch inmogint yme in keine wis von nuwem uffgeluhin werdin; waz hiewedir geschehe, daz hette keine krafft. $Z \mathrm{Z}$ i leste begriffin wir mit desem gesetze, daz die, von den die uffgabe nicht geschicht und weddir ir herrin frefelichin dun und sie wissinlichin anegriffint mit weddirsagin adir mit unwedirsagin ${ }^{5}$, zů stunt sint sie gefallin in alle vorgenant buße.

Das XV. capitel.

Von den bosen einhelligin, die sich vireynnegin wedir die, den sie billiche undirdan sint ${ }^{6}$.

Dye von den heiligin gesetzin virsmehetin und virwurffin eynunge und unzemeliche samenunge adir virbindunge in den

1 Or. De hiis, quibus ut indignis auferuntur bona feudalia. ${ }^{2}$ libere et realiter. $-{ }^{3}$ Hds. rede. $-{ }^{4} \mathrm{Hds}$. gegebin. - ${ }^{5}$ diffidacione obmissa. - Or. nur: De conspirationibus. 
stedin und uzwendig adir zusschin stat unde stat adir zusschin persone und stat adir zusschin persone und persone geslichtis ${ }^{1}$ halb adir wilchirleie andir schinis ${ }^{2}$; darzů globe ${ }^{3}$ und eit, virbintteniße und gedinge und ynbrachte gewonheit zů den selbin dingin, dye wir billichir schetzin ein stor ${ }^{4}$, virwerffin wir und virdampnen und von sichirm wissen virnichtin wirs als; welliche stede adir persone, ez sy undir in adir mit andirn, was wirdekeit ardis adir wesins sie sint, soliche virbinteniße bizher hant gedan adir hienach sich virmessen zu dunde ane irre herrin laube, der undirdan sie sint adir den sie sint dinstliche adir in der gebiede sie wonent, und die selbin herrin nit uznemen, alse die virbinteniße ane zwivel von den heiligen gesetzin der cristinlichir merir unsir furfarn sint virbotin und abegetan, als virbiedin auch wir und dun sie abe; alleine uzgenomen die fursichtekeit und virbintniße, die die fursten und stede undir einandir umb gemeinen frede der lande kuntliche undir in bestedeget hant, die behaltin wir sundirlichin unsir herluchtenunge und laßin sie blibin, biz daz wir zů rade werdin, waz wir davon ardenerin. Waz abir sundirlich persone furbaz me soliche globede virbinteniße bose vireinnunge und gedinge anefinge weddir dis geinwurtige gesetze und alt recht, daz davon gemacht ist, der fellit zů stunt in virlust siner erin und buße zehen phunt gultis; ist iz ein stat adir ein gemeine, die wedir daz gesetze dunt, die git hundirt phunt gultis und virliesin alle friheit und keisirliche wirdekeit; daz halbe deil der gultbuße fellit in keisirlichin sag ${ }^{5}$, daz andir deil dem herrin des landes, zů des schadin die virbinteniße geschehin ist; auch ${ }^{6}$ fallint die vorgenant die mynre nit in buße der altin recht, die weddir die bosen einhelligin gesetze sint.

Das XVI. capitel. Von den palburgirn.

Vil clage hat uns furbracht, daz etliche burgere und undirdan der fursten der landisherrin und andir lute suchint von in zů werffint die burdin geburtlichir undirdenekeit und smechlichin schaffin sie mit frefelichir gedurst, daz sie in andirn stedin inphangin werdin zu burgern, und hant daz

${ }^{1}$ pretextu parentele. - ${ }^{2}$ cuiuscunque coloris. $-{ }^{3} \mathrm{Hds}$. davor noch: dunt. $-{ }^{4}$ corruptela. $-{ }^{5}$ fiscus. $-{ }^{B}$ Dieser Satz im Or. schon vorher in kürzerer Fassung. 
hievor me gedan und nyt mynre ${ }^{1}$ wullin sie lipliche sitzin in landin stedin festin und dorffin der irstin herrin, die sie mit solichir geverde gelaßen hant, und der selbin stede fribeit, darin sye sich geburgert hant, wullint sie sich frauwen und hertriegint ${ }^{2}$ schyerm von in: sie heißin palburgir in Dutschem lande; herumb wan nů geverde und schalkeit nieman sulde zů helfe komen, so setzin wir uz sichirm wißin keysirlichiz gewaltis mit gesundim rade allir kurfurstin geistlichir und werntlichir und bestedegin mit desem gesetze zů ewigir werůnge, daz die vorgenant burgere und undirdan, die ir herrin also virspottint, in allin landin und stedin des beiligin richis von desem dage und hienach der stede friheit und recht nummer sullint genießin, in den sie sich in solichir geverde schaffint zi̊ burgern adir bizher geschaffint hant, ez sy dan, daz sie sich zů den selbin steden lipliche ziehin und darinne eygin hert stedeclichin habin und werlich ane driegin ${ }^{3}$ darinne wonnen und schuldigir burde und stede-sture in den selbin stedin undirdenig sin. Und welche weddir dis gesetze inphangin sint adir inphangin werdin, daz inphahin sal keine crafft han, und sullin noch inmogin in keinen sachin genießin fribeit adir recht der stede, da sie inphangen sin, ane weddirrede rechtis friheit adir gewonheit, von welchin zitin sie inthalten sin, die wir alle widdirruffin, alse vil alse sie desem gesetze widdir sint, und herkennen sie ane crafft umb die vorgenant stucke: alle die recht der furstin der herrin und andir lute, die sie hant an libe und an gude yrre undirdan, die sie in vorgenant wise laßin, sal in alle zyt behaltin sin. Und $\mathrm{me}^{4}$ [die], die vorgenant burgir und fromede ${ }^{5}$ undirdan weddir dis gesetze inphahin, laßin sie sie nit in eyme mande, nachdem daz dese geinwurtigen ding yn virkundit sint, so sint sie umb den selbin ubirgang gefallin in virlust hundirt mark lodegis gultis, alse dicke sie iz furbaz důnt; des gultis sal halb fallin in keisirlichin sag, daz andir sal ane abelaßin den herrin der lude, die weddir diz gesetze inphangin sint.

Das XVII. capitel. Von wedirsagen.

Alle, die sich furbaz me virmeßin wedir ieman von rechtir sache zů wedirsagen und den untzitlich in ir wonunge, adir

1 et nichilominus. $-{ }^{2}$ contendunt defensare. $-{ }^{3}$ non ficte. 4 Nichilominus hos. - 8 alienos. 
da sie gemeinliche nit wonen, weddirsagint, daz herluchtin wir, daz sie den wedirsagiten keynen schadin mit irin ${ }^{1}$ mogent gedun an brande an raube an zuckin ${ }^{2}$. Wan ${ }^{3}$ nů falsch" und geverde nieman sulde zů helfe komen, so bestedigen wir mit desem gesetze ewecliche zů werinde, daz die selbin weddirsagunge, die also geschehin ist adir hienach geschicht, nit sal crafft han wedir herrin adir andir lute, mit den die weddirsagere hant gewonit in geselleschafft in heimelichkeit adir in andir irlichir fintschafft; und sal auch nit sin, daz mit der wise eins iegelichins wedirsagins ieman angegriffin werde mit brande raube adir zuckin, ez sy dan, daz die wedirsagunge dry naturliche dage deme weddirsagetin vorhin lipliche virkundit werde uffinlichin adir an die stat, da he gewonlichen wonit, und daz von dem selbin virkunden mit waren gezugin sichir bekennen moge geschehin. Wer andirs ieman weddirsagit und ${ }^{5}$ anegriffit, der fellit zů stunt in unere, als ob kein weddirsagin were geschehin; und [wir] setzen auch, daz der selbe von eim iegelichim rittir als eyn virredir mit rechtir buße gepineget werde.

Wir virbiedin auch alle unrechte kriege und alle unrecht brant raub zuckin und unrecht zulle und unrecht geleide und abedrucken gewonlichis schetzins umb daz geleide undir der buße der heiligin gesetze, die ubir dye vorgenant ding undir ieclichis sundirlich zu bußen gesetzit sint.

\section{Daz XVIII. capitel.}

Von dem bryefe der kure an die kurfursten ${ }^{6}$.

"Uch hirluchten und großin furstin margrafen von Brannenburg des heiligin richis irtzkamerer unserme medekurfurstin und liebin frunde dun wir irtzbischoff von Mentze kunt myt desir geinwurtigen batschafft, daz die kure eins Romischin konigis sich zů dirre zit gebůrit zů dunde; und von schulde unsirs amptis so ruffin wir uch zů der selbin kure, alse wir sullin, daz ir von deme dage dis brieffis undir dren mandin nach einandir zu rechen von uch selbir mit uwirn geweltigin boten, eyme adir me, die adir der fullenkomen gewalt habin,

1 cum honore. - ${ }^{2}$ rapinas. - ${ }^{3}$ Hds. von; vgl, S. 118 A. $1 .-$ 4 fraus. - ${ }^{5}$ Hds. ane. - ${ }^{-}$Or. nur: Litera intimationis. 
komen zu der stat, die darzu gehorit nach der wise der heiligen gesetze, die darubir gemacht sint, alda zu beradin ubireinzůkomen mit andirn unsirn medekurfursten von der kur eins kunfftigen Romischin konigis, der danach mit godes helfe gefurderd werde zu eyme keisir, und auch an der selbin stat zů blibin, biz daz die selbe kure volbracht wird, und auch andir sachin zu dunde und uzzudragin, als man findet uzgeleit in den heiligin gesetzin, die darubir bedrechteclichin gemacht sint; und komen ir nit adir uwir botin, so furvarn wir doch uff ende mit unsirn medekurfursten in der vorgenant kur, alse die keisirlichin recht gesetzit hant."

\section{Daz XIX. capitel.}

Von der furme des gewaltbrieffis des kurfursten, der sine botin sendet $z$ ů der kůre.

„Wir Lodewig ${ }^{1}$ von gotz gnadin margrave zů Brannenburg din kunt allin gemeine mit dirre geinwurtigen schrifft, sit daz die kure eins Romischin konigis anfellit zů důne von redelichin sachen und wir mit sorgsame begerinde $\sin$ zů bedenckin umb des heiligen richis ere, daz izit schedelichin swerin schadin undirlege, so machen wir und setzin und ordenerin Johannem und Paulum ${ }^{2}$ unsir liebin und getrewin, von den wir sichirlichen sundir getrewin haltin ane allin zwivel, unsir ware rechtliche procurator und sundirbar badin (mit allin rechten wise und furme, in den wirs allir best und creftlichir mogin machin und kunen, sie beide und ieclichir ${ }^{3}$ in gantze macht, alse daz des recht und gedinge, der die sache undir handin hat, nit beßir sin, und waz von ir yme an wird gefangin, daz mag durch den andirn geendit werdin und recht-

${ }^{1}$ Entsprechend der in Cap. 18 an den Kurfürsten von Brandenburg gerichteten Aufforderung zur Wahl wird in unsrer Uebersetzung (im Or. allgemein gefasst: „Nos ... talis dei gracia etc.") in dieser Vollmacht der Kurfürst Ludwig d. Römer von Brandenburg († 17. Mai 1365) als Aussteller genannt; der Uebersetzer fingirt hier offenbar den zu seiner Zeit lebenden Kurfürsten von Brandenburg, welcher thatsächlich nie eine solche Vollmacht ausgestellt hat. Hatten wir früher (vgl. S. 128 A. 1) bereits das Jahr 1366 als möglichen Termin für die Abfassung unserer Uebersetzung gefunden, so ergiebt sich nun als Endtermin das erste Drittel des Jahres 1365. - ${ }^{2}$ Diese Namen sind fingirt; im Or. stehen dafür je drei Punkte. $-{ }^{3}$ sic! 
liche ende nemen,) zù dedingin an allin stedin mit andirn unsirn kurfursten geistlich und werntlichen und mit yn ubir einandir zů komen und zů enden umb ein wole, geschickete, ungebresthafte personen zu kiesin zů eyme Romischin konige, und daz die selbin procurator mogin geinwurtig sin den dedingin, zů habin ubir die kur solichir persone und zů handeln und bedrachtin, von unsirn wegin an unsir stat und namen die selben personen zů nennen und an sie zi gehellin und zu eyme Romischin konige zü furdirn und zu kiesen und uff unsir sele eynen ieclichin eit zi̊ sweren, der darzu notdurfftig ist adir gewonlichin wird, zů den vorgenant stuckin; und daz sie mogin eynen andirn adir andir scheftener setzin $\mathrm{zu}$ gantzir crafft und den selbin adir die selben zů weddirruffin und zu tůne alle ding, sundirlich waz zů den vorgeschrebin dingen notdurftig nůtze adir bequemeliche wirt zu dunde, geinwurtig zů sinde uff ein fulbrachtis ende des benennyns und des beradis der selbin kure und auch, ob die vorgenant stucke adir ir ieclichis heischin ein sundirlich gebot, auch ob die selbin groBir adir me sundirliche werin, dan die vorgenant ding adir wir selbir mochtin dun, obe wir dem gescheffte und der kunfftigen kur mit unsirs selbis libe geinwurtig werin; und wullin stede und geneme habin und globin festeclichin zü haltin ewecliche, waz durch die selbin vorgenant unsir scheffener und bodin und von den, die sie gesetzit hant adir setzinde sint, von in adir von ir yme in den vorgenant stucken gewirckit wirt adir geschicht, adir in wilchir wise daz geordenerit wirt."

Daz XX. capitel. Von der eynekeit der furstindum und recht, die in zugehorin et cetera.

Wan ${ }^{1}$ alle furstindum, von der craftit ${ }^{2}$ die werntlichen ${ }^{3}$ kurfursten recht und stimme habin an der kure eins Romischin konigis, mit dem selbin recht ampt und wirdekeit mit andirn rechten, die in zugehorint und davon hangint, also gar sint zusamen geschickit und unvirscheidenlichin vireynneget, daz recht stimme ampt wirdekeit, die zu iedem furstindum gehorit, nit gefallin mogin uff eynen andirn dan alleine an den; der daz furstindum mit lande und mit allim dem, daz darzu gehorit, kunt-

${ }^{1}$ Hds. von; vgl. S. 133 A.3. $-{ }^{2}$ quorum virtute. $-{ }^{3}$ Hds. ... chir. 
liche besitze mit dinstbirkeit mit lehin und hirschafft; daz bestedegin wir, daz eim ieclichin der vorgenant furstindum mit recht mit stimme der kure und ampt und mit allir wirdekeit, die darzu gehorint, also sin vireinet und ungedeilit by einandir blibin zi ewigin zitin, also daz der besitzir eins ieclichin furstindums sal sich frauwin eyner geruwigin frihin besitzunge recht stimme amptis wirdekeit und allir dinge, die darzu horint; und sal auch der selbe von allin kurfursten ein kurfurste gehaltin sin: der selbe unde nieman andirs sal von den kurfurstin zů allir zit zů der kure genomen werdin und zů andirn gedetin, die umb ere und nutz des heiligin richis sint zu důne, und ane alle weddirrede sal man in zulaßin; noch ir keins der vorgenant dinge sullint von einandir gedeilit werdin, wan sie sint und sullint unscheitbirlichin sin, noch inmag sie auch nieman zu deilin gefordirn weddir an gerichte adir uzwendig an gerichte noch mit urteil ubirkomen; ez sal auch nieman den horen, der da eins fordert ane daz andir. Geschehe iz abir von irůnge adir andirs, daz ieman virhort were, alle brieffe gerichte urteil, adir waz semelichis dingis davon uzginge weddir dese unse ordenunge, daz sal allis samt zů stunt mit keiner crafft besten.

Daz XXI. capitel. Von der ordenunge des furgangis der irtzebysch offe etc.

Wan ${ }^{1}$ wir da abin an deme drittin capitel zů rade wurdin fullinkommeclichin zů virsegin umb die ordenunge des sitzins undir den geistlichin kurfurstin in rade und zo dissche und andirs, alse dicke alse ${ }^{2}$ keisirlichir hoff gåhaltin wirt mit hirschafft adir wan sich furbaz me geburit, daz die kurfurstin mit dem keisir adir mit eyme Romischin konige gesamint sint, davon wir gehorit han; daz hievor krieg darumb gewesin ist, nu gleibin wir, daz iz gut sy, daz wir endin und uzlegin die ordenunge vorgendis undir in. Darumb so irkennen wir mit desem keisirlichim gebote, alse dicke in samenunge des keisirs adir eins Romischen konigis den vorgenant geistlichin kurfurstin mit dem keisir adir konige sich geburit zů gende und man keisirlich adir koniglich wapin fur yn dreit, so sal der

${ }^{1}$ Hds. von; vgl. S. 135 A. 1. $-{ }^{2}$ Hds. asse. 
irtzbischoff von Drere in glichir slechtir lynnegin gen vor dem keisir adir konige, und sal nieman zusschin in zwein gen, dan alleine die die keisirliche adir konigliche zeichin dragin; so abir der keisir adir konig ane die wapin get, so sal der selbe irtzbischof von Drere dem keisir adir konige vor gen, also daz nieman zusschin in zweyn sy; dye andirn zwene irtzbischof sullin ewicliche ir stede behaltin, als iz da abinan mit undirscheit irre provincien uzgeleit ist umb das sitzen.

\section{Daz XXII. capitel.}

Von der ordenunge des furgangis der werntlichen kurfurstin, und wir die keisirlichin und koniglichen wapin sulle keisir adir konige furdragen².

Uzzůlegen die ordenunge des furgangis der kurfursten, davon wir for rede han gehabit, so setzen wir, daz in der geinwurtikeit des keisirs adir des konigis so erget, alse dicke den kurfursten geburit zů gende nach der zile mit eim Romischin keisir adir konige: so ein uffinbar keisirlichir adir koniglichir hof ist, in waz sachin adir hirschafft daz sy, und man wirt dragin keisirliche gezirde, so sal der hirzauge von Sassin, der daz keisirliche adir konigliche swert dreit, vor dem keisir adir konige anemittil gen, und sal zusschin yme und dem bischoff von Drere niemant damittin sin; der paltzgrave sal den keisirlichin apil dragen zi der rechten siten; der margrave von Brannenburg sal daz ceptrum dragin zů der linketin siten ie weddir siten des hirtzaugen von Sassin; der konig von Beheim sal anemittel nach dem keisir gen.

\section{Daz XXIII. capitel.}

Von den segen der irtzbischoffe in der messe und zů dissche in keisirs und koniges geinwuitekeit etc. ${ }^{2}$

Alse dicke hirliche meße gehaltin wirt vor des keysirs adir Romischin koniges geinwurtekeit und den irtzbischofin von Mentze Drere und von Kollin adir ir zwein geinwurtig geburit zu sinde, sullin sie undir in dese ordenunge haltin:

1 Or.: De ordine processionis principum electorum et per quos insignia deportentur. - ${ }^{2}$ Or.: De benedictionibus archiepiscoporum in presentia imperatoris. 
in der bichte, die geschicht vor der messe, an dem herbieden daz ewangelige zů kossin, den freden nach dem ,agnus dei" umb zo̊ dragen und an den segin, die nach der messe und furre dissche zü thunde sint, und got zü lobin noch dissche in soliche wise des irsten dagis sal iz der irste allis důn, des andirn dagis der andir, des drittin dagis der dritte. Den irsten den andirn und den dritten bedůdin wir also zů virstende, nachdem daz ir ieglichir fưr adir noch zů buschoffe irwihit ist. Und daz eynre den andern mit wirdigir zemelichir ire fürkome und den andirn gut bilde gebin, sich undir einandir zů eren, so sal der, den der ordin rorit die vorgenant ding zi̊ dunde des dagis, den andirn mit eyme mynsamen nigin und winkin darzu ladin, und sal darnach furdreden zů den vorgenant dingen, alse iz sich danne heischicht.

\section{[II. Theil. Die Metzer Gesetze.]}

Daz XXIV. capitel.

Von den uffsetzigen weddir der kurfursten lip und lebin und der ufsetzigen buße und iren nachkomen und allir der, dye in $z$ ůgehorint ${ }^{1}$.

In ${ }^{2}$ deme hofe zu Metze, den wir keisir Karle der vierde Romischis und Beheimschis richis ein merer hiltyn in deme jare, da man zalte von Grist geburte druzehinhundirt jar und in dem sehsundfunftzigisten jare und by uns warent alle kurfurstin des heiligin richis mit geinwurtekeit der herrin des erwirdegen vatirs [Talayrand] bischoff zi̊ Albane der heiligin Romischin kirchin kardenal und Karle des konigis von Francrichis irste geborn son hirtzauge zu Normandi und delfin zu Fienne, in der herrin geginwurtekeit und zit, alse vor genant ist, so han wir die nach geschrebin gesetze gesagit uz.

[1] Wer mit furstin adir mit rittirn mit sundirlichin personen adir mit gemeyme fulke meyndedeclichin uffsatze dut adir mit eyden sich virbindit $z u$ dunde uff dot der erwirdegin des heiligin richis kurfurstin geistlichir adir werntlichir adir eyme undir in, der sal billich gebußit werdin; (die recht

1 Diese Ueberschrift findet sich in keiner der sog. Original - Handschriften der G. B. - ${ }^{2}$ Das klein Gedruckte ist ein späterer Zusatz, der als solcher im Kurmainzischen Exemplar kenntlich ist; in den Ausfertigungen für die Kurfürsten von Köln, Trier und der Pfalz fehlt dieser Zusatz, welcher eigentlich vor der Ueberschrift des Cap. 24 stehen müsste, gänzlich. 
wultint, daz wille der meyndat mit dem selbin grymme gepiniget wurde, alse die werk, und daz er mit dem selbin grimme gebußit wurde als ein schuldig man der almechtekeit, umb daz die vorgenant kurfurstin sint ein glet unsirs libis;) und sin gut in unsirn schatz geurteilit sy; [2] sine kint, den wir von keisirlichin gnaden virlihin daz lebin, sie sultin mit fedirlichir pine sterbin; daz bilde fedirlichir irbesunde sulte an in mit grymmer pine gemessin werdin: sie sullin virschaltin sin von mutirlichir zucht und von allim irbe irre nestin, und waz in von den mag gefallin, ensal auch nit fulgin selgerede von andirn luten; arm und gebrestin sullin sie eweclichin sin, fedirliche schande sal in alle zyt nachfulgin, zů keinen erin zu keiner werntlichir wirdekeit sullin sie nummer komen; sie sullin solich sin, daz in dot ein drost sy und daz lebin ein bittir pin, unde darin stinckende sin in ewigeme jamer; [3] danach heißin wir, [daz] alle die virlumunt sin und ane gnade, die fur soliche ummer virsuchint bede an uns zů legin. [4] Die dochtir, wie vil der ist, die sullin alle mit einandir nemen daz virde deil von der mudir gude, siege abe besetzit ${ }^{1}$ adir unbesetzit, uff daz sie habin mittelmeßige narunge billichir, wan daz sie gantzin nuitz adir irbe namen davon haltint; daz urteil sal miltir gein yn sin: wir getruwin, daz sie von krangheit frauwelichis ardis minre gedurstig ${ }^{2} \sin$. [5] Friheit, die sonen und dochtirn sint nach desem gesetze gebin, daz sie ledig sultin sin von fedirlichen undirdenekeit, komit in nit zů helfe. [6] Wir setzin auch, daz wedemegabe und viranderunge wilchirhande gude, mit wilchir geferde adir recht man findet, daz sie geschehin sint von der zyt, da die vorgenant zum irsten gedachtin den uffisatz und geselleschaft anzůgriffin, daz sal keine krafft han. [7] Der vorgenant uffsetzigin husfrauwin, die irin wedemen herkobirt hant, und die gut, die sie von irrin mannen inphangin hant in giftis recht, sint die in dem gedinge, daz sie den sonen sullint gefallin zu der zyt, so die $^{3}$ schare ${ }^{4}$ ist abegenutzit, so sullin sie wißin, daz alle die gut, die den sonen nach dem gedinge sultint werdin, in unsen keisirlichin schatz horint; [8] doch sal den dochtirn von den selbin gutin daz vierde deil werdin und nit den sonen.

1 sive testata sive intestata. - ${ }^{2}$ minus ausuras. - ${ }^{3}$ Hds. sie. - ${ }^{4}$ ususfructus (Ertrag, Einkünfte). 
[9] Andir werbe waz wir von den vorgenant und iren kinden gesetzit han, daz wullin wir in hirtekeit gehalten han an irin knechtin an irin dienern, die der missetat midewissinde sint, und der selbin kinde. [10] Wer abir undir den von ursprunge der anegefangin missetat mit fliße eins waren labis die meindat meldit, der sal mit lone und mit erin von uns begabit werdin; abir der an dem ufflegin schuldig wirt, ob der doch undir unkuntlichir wise die heimelichkeit der rede uffinbarit, der sal losunge und gnadin wirdig sin. [11] Andir ${ }^{l}$ werbe umb die selbin missetat, die sich driffit zu geletztir almechtekeit an den kurfurstin, sullint auch die knechte an des herrin houbit gepiniget werdin. [12] Hernach setzen wir, ob it uffsatzis geschehe weddir die vorgenant kurfurstin geistlichin und werntlichin und missetan wurde, daz auch noch dote des schuldegen daz lastir mag hernnuwit werdin. [13] Darzu bestedegin wir mit desem gebode, daz nach dote der schuldegen die meyndat moge beclagit werden, und des ubirwundin dotis gehugnißis sal man virdampnen und sin gut sal man den irben abenemen; [14] wan von dem, daz eynre ein meyndedegin rat [fassit], so ist er zů stunt etlichir maße gepinget an sime gedancke; darzi bekennen wir mit deme, daz einre al soliche missetat an sich zuhit, daz der nit viruzsirn adir nieman fri von dinstbirkeit gelaßin mag, und yme sin schuldener von rechte nit geldin sal. [15] In der sache setzin wir, daz die knechte gepiniget werden den herrin zu leidin, daz ist umb die sache des virdamptin uffsatzis weddir die kurfurstin, alse vor geseit ist. [16] Und ob einre sturbe, daz nachkomen gut sal gehaltin werdin von der ingewollin ${ }^{2}$ persone halb, ob er alse dot gewisit wirt, daz er gewesen ist in der selbin sache.

\section{Daz XXV. capitel.}

Von den nachkomen der werntlichin kurfursten ${ }^{3}$.

$\mathrm{Ez}$ bekomit wol, daz andir furstindum by einandir gantz blibet, daz gerechtekeit gestirkit werde und die getruwin undirdan sich frauwin ruwe und frede: nichils me sullint die grossin furstindum birschafft ere unde recht der kurfursten

${ }^{1}$ Die $\$ \S 11$ und 12 im Or. umgestellt. - ${ }^{2}$ incerta. - ${ }^{3}$ In den Or.- Hdss. keine Ueberschrift. 
ungeletzit by einandir blibin, wan da me schadin lit, da sal man helfe zulegin, so die sule fallin, daz die weddirsetze des buwis nit zusamen slahe; herumb stedegin wir und bekennen mit gebode ewige zit zů werin, daz von nů furbaz me die edeln und grossin furstindum, daz rich von Beheim, paltzgraveschafft des Rines, hirtzaugedum von Sassin, margrafedum von Brannenburg, ir lant gebiede manschaft' dinstbirkeit, und waz andirs darzu gehorit, sullint nummer virsnidin adir virdeilit werdin, me sie sullint billichir ewecliche blibin in irrim fullinkomen gantzheit. Der irste geborin son sal an den hirschefftin und rechten irben ${ }^{1}$ : der horit alleine darzu, er were dan nit by sinnen adir ein dor adir eins virmertin ${ }^{2}$ mirglichis gebrestin, von des wegin er nit sulte noch inmochte ubir die lude nit ein furste gesiu; in der geschichte sal der andir geborin son irben, ob er ist in deme geslichte, adir darnach der andir alste brudir adir leies mag, der von fedirlichim stamme zi dem nestin ist abekomen in rechtir lenige. Der selbe sal sich zu hant gein den andirn brudirn und swestirn minsame und gnedeclichin herbiedin nach der gnadin, also yme got git nach sime wolegefallin, und nach irbem macht, also umb in alle wise virbodin sy deilin unde intledin furstindum und allis, daz darzu horit.

\section{Das XXVI. capitel.}

Wie die kurfurstin von keisirlichem hofe sullent komen ${ }^{3}$.

[1] An deme dage, so keisirlichir adir koniglichir hof in wirdekeit gehaltin wirt, so sullin die kurfurstin alle komen zů prime zit zu des keisirs adir konigis hirburge, und sal der keisir adir der konig angedan sin mit allir keisirlichir wede ${ }^{4}$, und ieder kurfurste uff sinem perde sullint mit deme keisir riten an die stat, da man sitzin sal: ir ieclichir sal gen in ordenunge und wise, alse iz vor gesetzit ist des furgangis der kurfursten und fullinkommeclich ist uzgedragin. Der irtzkantzelir, in des irtzkantzelerie der hof gehaltin wird, sal brengin uff eyme silbirn stabe alle ingesigel unde keisirliche adir konigliche briefe-zeichen; die werntlichin kurfurstin sullint

${ }^{1}$ Hds. noch: irbin. $-{ }^{2}$ famosi et notabilis defectus. $-{ }^{3}$ In den Or.-Hdss. keine Ueberschrift. - ${ }^{\circ}$ Oben (s. S. 110 A. 1) wat. 
brengen daz ceptrum den apil und daz swert; zum irstin sal man auch dragin vor deme irtzbischofe von Drere anemittel, so er fur get in siner ordenunge, zum irstin die krone von Ache, darnach die krone von Meilon von etlichin nedirn furstin, die der keisir adir der konig darzu schickit; daz dragen mit den kronen sal alleine geschehin deme, der mit keisirlichen adir mit koniglichir imfeln gezerit ist.

[2] Die keisirynnen adir die Romische koniginnen sal mit irre keisirlichen adir koniglichir wede gecleidet zogin zů der sitzestat, von irren großin herrin geselgit mit yren nachfulgerin juncfrauwin etwie ferre eyn zemeliche wide nach deme keisir adir Romischen konige und auch nach deme konige von Beheim, der dem keisir allirneste nachfulgit.

\section{Daz XXVII. capitel.}

Von den ampten der kurfursten in keisirlichem adir koniglichem hofe.

Wir setzin, wan der keisir adir Romisch konig uffin hoff heldit, da die kurfurstin mit yrin amptin sullint dienen, so sal die nachgeschrebin ordenunge gebaltin werdin:

[1] Zům irstin, so der keisir adir der konig sitzit uf sime keisirlichin adir koniglichim trone, so sal der hirtzauge von Sassin sin ampt dun in dese wise: man sal schudin for daz keisirliche gesesse ein huffen habirn, der also hoch sy, daz er dem perde ge an die brust adir an den vordern reiff; uff dem perde sal der hirtzauge sitzin und sal han eyne silbirn strichin in siner hant und ein silbirn summern ${ }^{1}$, die beyde sullint han an gewiechte 12 marck silbirs; und also sitzint uf dem perde sal er daz mas ful habirn nemen und sal den dem irstin knechte, der da komit, schudin in sinen sag. So er daz gedut, so sal er die strichin steckin in den habirn und sal abezogen, und sin undirmarschalg der von Pappinberg ${ }^{2}$ der sal an sine stat adir des hofis marschalg, obe der von Pappinberg nit da inwere, den habirn furbaz uzmessin. So der keisir adir der konig inget zi̊ dissche, so sullin die geistlichin kurfursten irtzbischhofe sten for dem dissche mit andirn prelatin unde sullint den disch gesegin nach der ordenunge, alse daruff

1 = sumber, Korb; Or. mensura. - ${ }^{2}$ sic! später (S. 146) richtig. 
vor geschrebin ist; so der disch gesegint ist, so sullint die selbin irtzbischofe, obe sie da sint, adir zwene adir eynre die ingesigel und keisirliche adir konigliche briefe-zeichin von deme bofekantzilir nemen an deme silbirn stabe, und der irtzbischof, in des kantzelerie der hof gehalten wirt, sal mittin gen und die zwene ie wedir sit neben sit und sullint alle den stap, da die ingesigel anehangin, mit irin [handin] anegriffin und sullin sie dragin und wirdeclichin legin fur den keisir adir konig uf den disch; der keisir adir konig sal sie in zů stunt weddirgebin, und der irtzbischoff, in des kantzelarie daz geschicht, der sal daz große ingesigel dragin an sime halse, bis daz man gessin hat, und darnach bit daz er komit an sine hirburge von keisirlichim adir koniglichem hafe [!] geredin. Der stap, da die ingesigel anehangin, sal von 12 mark silbirs sin; des silbirs sal ieder irtzbischof gebin daz dritte deil; der stap mit den ingesigeln und briefe-zeichin sal zůgeantwurtit werdin des hofis kantzelir in sinen nuitz zů kerin, wie iz yme selbir fugit. Und nachdem daz der irtzbischof, dem daz große ingesigel geburit zü dragin, komit von keisirlichem hofe in sine hirburge, so sal he daz selbe ingesigel sendin mit eyme sinis gesindes uff eime perde deme hofekantzlir; daz pherd ist her schuldig zů gebin dem selbin kantzilir nach zemelichkeit siner eigin wirdekeit und minne, die er zi yme hat.

[2] Der margrafe von Brannenburg sal komen uff eyme perde und sal han zwei silbirn beckin mit waßir in den hendin und eine schone hantwehil und sal sitzin von sime pherde und sal deme keisir adir deme konige waßir gebin ubir die hende: die beckin sullint halten 12 mark silbirs.

[3] Der paltzgrave von Rine sal inriten uf eyme perde mit vir silbirn schusselin in sinen hendin, die sal iecliche haltin 3 mark silbirs, und sal abesten von dem perde und sal die schußelin setzin uf den disch fur den keisir adir konig.

[4] Darnach sal der konig von Beheim komen uf eyme pherde und sal brengen eynen silbirn kop von 12 marck silbirs mit wine und mit waßir gemisschit und sal sten von sime pherde und sal den kop dem keysir adir konige zi drinckin biedin.

[5] Alse wir funden han, daz bizher gehaltin ist, also setzin wir auch, so die werntlichin kurfursten ir ampt han 
getan, so sal der von Falkinstein ein fitzkamerer des richis zů eme nemen pherde und beckin des marggrafin von Brannenburg; der kochinmeistir von Norttinberg sal nemen pherde und schußelin des paltzgrafen; der schencke von Limpburg sal nemen pherde und kop des konigis von Beheim; der von Pappinberg ${ }^{1}$ der sal nemen pherde unde stap und summern des hirtzaugin von Sassin, ob die selbin sint zůgegin und ir iegelichir dinte yn sime ampte; ist abir, daz ir etlichir in deme hofe nit geinwurtig were, so sullin des keisirs adir des konigis dageliche hofediener, nachdem als iegelichir gemeinsamt an dem amte mit dem, der nit zůgegin ist, als er daz ampt dreit, also sal er auch den nutz ufhebin.

Das XXVIII. capitel.

Von der ordenunge der dische in dem keisirlichin adir koniglichem of fen hofe ${ }^{2}$.

[1] Keisirlichir adir koniglichir disch sal also gefugit sin, daz er ubir andir dische in dem sal sehs fuße hohir sy gesetzit, an dem nieman sal sitzin in dem dage des hirlichin hofis dan alleine der keisir adir konig.

[2] Der stul und der disch der keisirynnen adir konigynnen sal bereit sin in dem sal, also daz ir disch dry fuße nedir sy dan des keisirs adir konigis und dri fuße hohir dan der kurfurstin disch; die kurfurstin sullen undir in in glichir hohe stule und dische han.

[3] Undir des keisirs gese ße sins dischis sal man bereiten den selbin kurfurstin geistlichin und werntlichen zů der rechtin hant dry, zo̊ der linketin hant dry geseßin; daz sebinde geseße sal man bereitin glich gein des keisirs adir konigis antlitze, als iz davor von dem sitzin der kurfurstin uzgesprochin ist; auch also daz nieman andirs, waz wirdekeit er ist, sitzin sal undir in adir zů den dischin.

[4] Iz zemit auch nit den werntlichin kurfurstin, daz ir eynre sich setze zů sime dische, diewile der andir einre sin mitkurfurste sin amt noch dan zi dunde hat; wan so ir einre sin ampt fullinbracht hat, so sal er gen zů dem dische, der

1 S. auch S. 142 A. 2. - 2 Ueberschrift fehlt im Or. 
eme bereit ist, und sal daby stende beidin ${ }^{1}$, bis daz die andirn ir ampt hant gedan; danach sullin sie sich alle und sundir mit einandir setzin $z \mathfrak{u}$ den disschin, als in ir ieclichim bereit ist.

$$
\text { [Das XXIX. capitel.] }{ }^{2}
$$

[1] Wir han daz auch fundin von warir sage und gesetze der altin, von der zit itzunt nieman andirs gedenckit, daz von unsirn seligin furfarin flißeclich gehaltin ist, daz eins Romischin konigis kure zi̊ Frankinfurd in der stat geschehin sal, zů Ache gecronit werdin, sin irsten hof zi̊ Nurmberg haltin; darumb wullin wir von sichirn sachin, daz die selbin ding in kunftigin zitin gehaltin werdin, ez inwere dan, daz den vorgenant stuckin allin adir etlichen redeliche hindirniße begenten. [2] Wan iz auch geschehe, daz ein kurfurste geistlich adir werntlich mit rechtim hindirniße geirrit werde, so er geheischin were zů dem keisirlichin hofe, und nit komen mochte, der bode adir procurator, den er sendit und den man zů sal laßin, nach dem alse sin gebot stet, waz wirdekeit adir wesins er ist, so sal er doch nit $\mathrm{zu}$ dische adir zů stule sitzin, alse dem bereit ist, der in gesant hat.

[3] So nů alle ding fullinbracht sint, die zu keysirlichim adir zu koniglichim hofe zu richtin sint, so sal der hofemeistir zu eme nemen allin bi keisirlichis adir koniglichis geseßis, da keisir adir konig geseßen ist, mit den kurfurstin den hof zů halten adir lehin zů lihin, alse vor uzgegebin ist.

\section{Daz XXX. ${ }^{3}$ capitel. Von den furstin, die lehin inphahint, waz sie gebin sullint et cetera 4}

[1] Wir setzin mit desem keisirlichin gesetze, daz die kurfurstin geistlichin und werntlichin, so sie konigliche lehin von keisir adir von konige inphahint, so sullin sie nieman gebundin sin icht zu gebin; wan daz gelt, daz in solichir wise gegebin wurde, daz hurte den amptluden zů. Wan ${ }^{5}$ nů alle kurfursten in keisirlichem hofe ubir alle ampte sin, wan sie kamerer kochinmeistir schencke und marstellir sint und hant

1 beiten = warten (exspectare). $-{ }^{2}$ Diese Ueberschrift (Abgrenzung) fehlt in der Hds. $-{ }^{3}$ Hds. XXIX, vgl. A. 2. -4 Or.: De iuribus officialium, dum principes feuda sua ab imperatore vel rege Romanorum recipiunt. $-{ }^{5}$ Hs. von; vgl. S. 136 A. 1. 
undir in in den selbin amptin gesetzte undirdan, die ime von Romischin fursten gegebin und gewedemit sint, darumb were iz ein daube rede, daz die undirdane amptlude von irin obirstin amptherrin, und in wilchir wise daz geschehe, [geschenke furdern], ez were dan villichte, das die selbin kurfurstin irin undirn amptluden etwaz frilich und willeclich wultin gebin.

[2] Abir dye andir furstin des richis geistlich adir werntlich, so ir eynre lehin inphehit von Romischin keisir adir konige, der sal gebin den amptludin in des keisirs adir konigis hofe 63 mark silbirs und eynen firdung, ez sy dan, daz ir einre mit friheit adir mit keisirlichir adir mit koniglichir laube sich beschirmen moge und bewerin, daz er ledig uzgenomen sy von solichir gulte und auch von andirn dingen, die von gewonheit sint zů geltin umb soliche inphahin der lehin. Die vorgenant 63 marck und firdung silbirs sal der hofemeistir des keisirs adir konigis also deilin: er sal eme selbir zum irstin behaltin 10 mark, dem kantzelir zehin mark, den meistirn den schribirn den dicterern 3 mark, dem ingesigelir umb daz waz und birmynt den eynen firdung, also daz der kantzelir und die schribir dem furstin, der daz lehin inphangen hat, nit andirs sint schuldig zů gebin dan gezug-brieffe des inphangen lehins adir eynen brieff eyner eynfeltigin inleidunge.

[3] Auch sal der hofemeistir gebin dem schenkin von Limpburg 10 mark, dem kochinmeistir von Norttinberg 10 mark, dem undirmarschalke von Pappinheim 10 mark, dem kemmerer von Falkinstein 10 mark mit solichin gedingen, ob sie und ir iegelichir geinwurtig sint in den hohen hofen und iegelichir dinte sime ampte; were abir, daz die vorgenant undiramptlude adir ir iegelichir nit geinwurtig weren, so sullint die amptlude des keisirs adir konigis, die ubir solich ampt sint, der abesint, der stat sie virwesint, in des amptis namen und arbeit er dreit, also sal er nutz und gewin nemen.

[4] So auch ein furste lehin inphehit von keisir adir von konige und uf eyme pherde adir uf eyme andirn fiehe sitzit, daz phert adir daz fiehe, willichirleie iz ist, sal deme obirstin marschalke deme hirtzaugin von Sassin werdin, ob er geinwurtig ist; andirs iz wurde dem undirmarschalke von Pappinheim: ist er nit da, so wirt iz des keisirs adir konigis hofemarschalg. 


\section{Daz XXXI. ${ }^{1}$ capitel.}

Von den manigerhande zungen der kurfursten ${ }^{2}$.

Sit daz die wirdekeit Romischis richis hat zů bedrachtin gesetze unde uzrichtin manigirhande lande, die undirscheidin sint an $\operatorname{setin}^{3}$ an leben an sprache, so ist von urteil allir wise wirdig und nutze geachtit, daz die kurfursten, die da sint sule und gledir des richis, sin gelerit in undirscheit manigirhande sprache unde zungen, daz sie fele lude virsten, und auch daz man sie virste, und daz sie mit sorgsamkeit vil lutin ir not uffhebin; darumb setzin wir, daz der herluchtin kurfursten des konigis von Beheim, des paltzgraven ${ }^{4}$ von Rine, des hirtzzaugin von Sassin und des margraven von Brannenburg sone adir ir irbin adir ir nochkomen gelerit werdin in Latiner Lamparter und Slefener zůnge, anzifahin von deme jare irs altirs, wan iz dofur zii haltin ist, daz in Dutsche sprache zů wißin naturlich ingeplantzt ist von kintheit zů lerin, also daz sie undir $14^{5}$ jarin irs altirs gelerit sin in solichin zungen, alse sie dan gnade von gode darubir han inphangin; daz wirt nit alleine nutze, iz wirt auch von vorgenant sachin gar hohe notdurftig gehaltin. Darumb daz die zungen dicke zů nutze und notdurft des heiligin richis gewonlich sint zi̊ ubin und in strengen sachin des richis me virjagit ${ }^{6}$ werden dese wise zů lernen, setzin wir also $\mathrm{zu}$ halten, daz iz blibe an fedir wille, ob sie sone hant adir ire nestin, von den sie sich virsehin, daz sie irben an den furstendum, schickin zi̊ den stedin, da sie die selbin zungen mogen lernen adir in zů fugin in irin eygin husirn zuchtmeistir lerer und kinde-medegesellin, die auch darinne virstendig sint, mit der wandele und lere sie mit einandir mogint in den zungin undirwisit werden.

${ }^{1}$ Hds. XXX; vgl. S. 145 A. 2. - ${ }^{2}$ Ueberschrift fehlt im Or. ${ }^{3}$ sic! - ${ }^{4}$ Hds. der paltzgrave. - ${ }^{5}$ Hds. XXIV! - ${ }^{6}$ ventilantur. 